\title{
Proof of the symmetry of the off-diagonal heat-kernel and Hadamard's expansion coefficients in general $C^{\infty}$ Riemannian manifolds
}

\author{
Valter Moretti \\ Department of Mathematics, Trento University, and Istituto Nazionale di Fisica Nucleare, \\ Gruppo Collegato di Trento, I-38050 Povo (TN), Italy. \\ E-mail: moretti@science.unitn.it
}

February 1999

\begin{abstract}
We consider the problem of the symmetry of the off-diagonal heat-kernel coefficients as well as the coefficients corresponding to the short-distance-divergent part of the Hadamard expansion in general smooth (analytic or not) manifolds. The requirement of such a symmetry played a central rôle in the theory of the point-splitting one-loop renormalization of the stress tensor in either Riemannian or Lorentzian manifolds. Actually, the symmetry of these coefficients has been assumed as a hypothesis in several papers concerning these issues without an explicit proof. The difficulty of a direct proof is related to the fact that the considered off-diagonal heat-kernel expansion, also in the Riemannian case, in principle, may be not a proper asymptotic expansion. On the other hand, direct computations of the off-diagonal heat-kernel coefficients are impossibly difficult in nontrivial cases and thus no case is known in the literature where the symmetry does not hold. By approximating $C^{\infty}$ metrics with analytic metrics in common (totally normal) geodesically convex neighborhoods, it is rigorously proven that, in general $C^{\infty}$ Riemannian manifolds, any point admits a geodesically convex neighborhood where the off-diagonal heat-kernel coefficients, as well as the relevant Hadamard's expansion coefficients, are symmetric functions of the two arguments.
\end{abstract}

\section{Introduction.}

After earlier works (e.g. see Wa78]), the symmetry of the coefficients which appear in the short-distance-divergent part of the Hadamard expansion of the two-point functions of a quantum state in curved spacetime, has been tacitly assumed to hold in mathematicalphysics literature. This symmetry plays a central rôle in the renormalization procedure of the one-loop stress tensor in curved spacetime, either in Lorentzian and Euclidean Quantum Field Theory. In fact, it is directly related to the conservation of the 
stress tensor and the appearance of the conformal anomaly [Wa78]. On the other hand, the symmetry of Hadamard coefficients is related to that of the heat-kernel coefficients BDD82, Fu91, Mo98a, Mo98b. Despite the relevance of this assumption, to the knowledge of the author, up to now no rigorous proof of these symmetries exists in the literature 1 . In this paper, we shall see that the problem of the symmetry of the heat-kernel/Hadamard coefficients is not so trivial as it seems at first sight. That is related to the fact that, in principle, the heat-kernel expansion could be not asymptotic in the rigorous sense, also in the Riemannian case, when it is performed off-diagonal. We shall prove that the heat-kernel coefficients, in the Riemannian case, are actually symmetric in a geodesically convex neighborhood of any point of a $C^{\infty}$ manifold. As a result we shall also see that the requirement of analyticity of the manifold assumed in earlier work Wa78, BO86] can be completely dropped (as argued in [FSW78).

\section{Generalities, the problem of the symmetry of the heat-kernel and Hadamard's coefficients}

1.1 Notations, general hypoteses and preliminaries. Within this work, $\mathcal{M}$ denotes a (Hausdorff, paracompact, connected, orientable) $D$-dimensional $C^{\infty}$ manifold endowed with a non-singular metric $\mathrm{g}$ which makes $\mathcal{M}$ geodesically complete. $\mathcal{M}$ can be a manifold with smooth boundary $\partial \mathcal{M}$ and we shall consider $\mathbf{g} \equiv g_{a b}$ either Lorentzian or Riemannian.

We shall deal with differential operators of the form

$$
A_{0}=-\Delta+V: C_{0}^{\infty}(\mathcal{M}) \rightarrow L^{2}\left(\mathcal{M}, d \mu_{g}\right),
$$

if $\mathcal{M}$ is Riemannian, and

$$
A_{0}=-\Delta+V: D(\mathcal{M}) \rightarrow C^{\infty}(\mathcal{M}),
$$

if $\mathcal{M}$ is Lorentzian. $D(\mathcal{M})$ is any domain of smooth functions, like $C_{0}^{\infty}(\mathcal{M})$ or $C^{\infty}(\mathcal{M})$. $\Delta:=\nabla_{a} \nabla^{a}$ denotes the Laplace-Beltrami operator and $\nabla$ means the covariant derivative associated to the metric connection. $d \mu_{g}$ denotes the Borel measure induced by the metric, and $V$ is a real function of $C^{\infty}(\mathcal{M})$. Whenever $\mathcal{M}$ is Riemannian, we suppose also that $A_{0}$ is positive, namely, that it is bounded below by some constant $C \geq 0$ (for sufficient conditions for this requirement see Da89]).

The requirents above are the general hypotheses which we shall refer to throughout this paper.

\footnotetext{
${ }^{1}$ For instance, in Wa78, such a symmetry was (indirectly) argued to hold for the analytic case. In [FSW78], the symmetry was argued to hold for the $C^{\infty}$ case. Neverthenless, these papers did not report the corresponding proof. The literature concerning the point-splitting procedure successive to [Wa78], as [BO86], assumes that symmetry implicitly.
} 
Let us give some definitions used throughout this work and recall some known and useful relevant results.

In this paper, a manifold with boundary $(\mathcal{M}, \partial \mathcal{M})$ is defined by giving a pair $\left(\mathcal{N}_{\mathcal{M}}, f_{\mathcal{M}}\right)$, where $\mathcal{N}_{\mathcal{M}}$ is a manifold and $f_{\mathcal{M}}: \mathcal{N} \rightarrow \mathbb{R}$ denotes a differentiable function. The set $\mathcal{M}$ is defined by $\mathcal{M}:=\left\{p \in \mathcal{N}_{\mathcal{M}} \mid f_{\mathcal{M}}(p) \geq 0\right\}$ and the boundary $\partial \mathcal{M}$ is defined by $\partial \mathcal{M}:=\left\{p \in \mathcal{N}_{\mathcal{M}} \mid f_{\mathcal{M}}(p)=0\right\}$.

Throughout the paper an analytic function is a real-valued function which admits a (multivariable) Taylor expansion in a neighborhood of any point of its domain. Moreover, "smooth" means $C^{\infty}$ whenever we do not specify further.

In the Riemannian case, $A_{0}$ is symmetric and admits self-adjoint extensions Mo98a. In particular, following the spirit of Wa78 and Mo98a, Mo98b], at least in the case $\partial \mathcal{M}=\emptyset$ we shall deal with the Friedrichs extension [त्RS80] which will be denoted by $A$ throughout this paper. We remind that $A$ has the same lower bound than $A_{0}$. Moreover $A_{0}$ is essentially self-adjoint and thus $A$ is its unique self-adjoint extension whenever either $\mathcal{M}$ is compact Mo98a or $V \equiv 0$ Da89.

Concerning derivative operators, we shall employ the following notations, in a fixed local coordinate system,

$$
D_{x}^{\alpha}:=\left.\frac{\partial^{|\alpha|}}{\partial x^{1 \alpha_{1}} \cdots \partial x^{D \alpha_{D}}}\right|_{x}
$$

where the multi-index $\alpha$ is defined by $\alpha:=\left(\alpha_{1}, \cdots, \alpha_{D}\right)$, any $\alpha_{i} \geq 0$ being a natural number $(i=1, \cdots, D)$ and $|\alpha|:=\alpha_{1}+\cdots+\alpha_{D}$.

Whenever $I \subset \mathcal{N}$ is a closed subset of the manifold $\mathcal{N}, f \in C^{k}\left(I ; \mathbb{R}^{n}\right)$ indicates a $\mathbb{R}^{n}$-valued function defined on $I$ which admits a $C^{k}$ extension on some open set $I^{\prime} \subset \mathcal{N}$ such that $I \subset I^{\prime}$.

Finally, in a fixed coordinate system, $\nabla f$ indicates the function which maps any point $q$ to the Jacobian matrix evaluated at $q$ of the function $f: p \mapsto f(p)$.

In any manifold $\mathcal{M}$ endowed with a (not necessarily metric) affine connection which makes it geodesically complete, the notion of normal neighborhood centered on a point $p, \mathcal{N}_{p}$, indicates any open neighborhood of the point $p \in \mathcal{M}$ of the form $\mathcal{N}_{p}=\exp _{p}(B)$, $B \subset T_{p}(\mathcal{M})$ being an open starshaped neighborhood of the origin such that $\exp _{p}$ defines a diffeomorphism therein. Then, the components of the vectors $v \in T_{p}(\mathcal{M})$ contained in $B$ define normal coordinates on $\mathcal{M}$ centered in $p$ via the function $v \mapsto \exp _{p} v$. Notice that any $q \in \mathcal{N}_{p}$ can be connected with $p$ by only one geodesic segment completely contained in $\mathcal{N}_{p}$. It minimize the length of the class of curves connecting these two points when the connection is metric, the metric is Riemannian and $B$ is a geodesical ball. A totally normal neighborhood of a point $p \in \mathcal{M}$ is a neighborhood of $p, \mathcal{V}_{p} \subset \mathcal{M}$, such that, for any $q \in \mathcal{V}_{p}$, there is a normal neighborhood centered on $q$ containing $\mathcal{V}_{p}$. Therefore, if $q$

\footnotetext{
${ }^{2}$ In this work, a neighborhood of a point is any set which includes an open set containing the point.
} 
and $q^{\prime}$ belong to the same totally normal neighborhood, there is only one geodesic segment connecting these two points completely contained in any normal neighborhood sufficiently large centered on each of the points (but this segment is not necessarily contained in $\mathcal{V}_{p}$ ). Notice that a coordinate system which covers any totally normal neighborhood does exist in any case: It is that defined in a sufficiently large normal neighborhood of one of its points. Finally, a geodesically convex neighborhood of a point $p \in \mathcal{M}$ is a totally normal neighborhood of $p, \mathcal{U}_{p}$, such that, for any pair $q, q^{\prime} \in \mathcal{U}_{p}$, there is only one geodesic segment which is completely contained in $\mathcal{U}_{p}$ and connects $q$ with $q^{\prime}$.

Statements and proofs of existence of normal, totally normal and convex neighborhoods of any point of any geodesically complete manifold can be found in [KN63] for affine connections and dC92, BEE96 for the Riemannian and Lorentzian case respectively. If a (complete) Riemannian manifold has an injectivity radius $r>0$ dC92, then each pair of points $p, q$ with $d(p, q)<r$ is contained in a totally normal neighborhood.

If $\mathcal{M}$ admits a boundary, all the definitions above and results concerning normal, totally normal and convex neighborhoods of points away from the boundary hold true.

1.2 Heat-kernel coefficients, Hadamard parametrix and the problem of the symmetry in the arguments. In this part we discuss informally some features of heat-kernel coefficients in both the Lorentzian and the Riemannian case.

In our general hypotheses on the manifold $\mathcal{M}$ endowed with the metric $\mathbf{g}$, fixing any open totally normal (or geodesically convex) neighborhood $\mathcal{N}$, the so-called world function is defined, for $(x, y) \in \mathcal{N} \times \mathcal{N}$, as the real-valued map

$$
(x, y) \mapsto \sigma(x, y):=\frac{1}{2} \mathbf{g}(x)\left(\exp _{x}^{-1}(y), \exp _{x}^{-1}(y)\right)\left(=\frac{1}{2} \mathbf{g}(y)\left(\exp _{y}^{-1}(x), \exp _{y}^{-1}(x)\right)\right) .
$$

$\sigma(x, y)$ does not depend on the chosen particular open totally normal neighborhood which contains $x$ and $y$. As is well-known [KN63, BEE96, dC92], $\sigma$ is always smooth in $(x, y)$ and furthermore analytic in $x$ and $y$ (separately in general) whenever the metric is analytic. This is because, in open totally normal neighborhoods, the function $(x, y) \mapsto \exp _{x}^{-1}(y)$ is always $(x, y)-C^{\infty}$ or analytic in $x$ and $y$ KN63 if the metric is so. Moreover, whenever the metric is Riemannian and the manifold has an injectivity radius $r>0$ (this holds for compact manifolds in particular), $\sigma$ can be defined on its natural domain $\mathcal{D}_{r}:=$ $\{p, q \in \mathcal{M} \mid d(p, q)<r\}, d$ being the Riemannian distance on the manifold. Indeed, in the considered situation, $\sigma$ belongs to $C^{\infty}(\{p, q \in \mathcal{M} \mid d(p, q)<r\})$. This is because of Sobolev's Lemma [Ru97, since, in the Riemannian case, the function $(x, y) \mapsto \sigma(x, y)=$ $d^{2}(x, y) / 2$ is continuous everywhere on $\mathcal{M} \times \mathcal{M}$ and is smooth in each variable separately on $\mathcal{D}_{r}$.

We pass to summarize the main features of the "small $t$ expansion" of the heat kernel $K(t ; x, y)$ of the positive operator $A_{0}$ in compact Riemannian manifolds supposing that our general hypotheses are fulfilled [Ch84, Gi84, Sh87, Da89, Ca90, Fu91, Ta96, Mo98a]. The heat kernel is the integral kernel of the semigroup of positive self-adjoint operators 
$\left.e^{-t A}, t \in\right] 0,+\infty\left[\right.$ which is a solution in $C^{\infty}((0,+\infty) \times \mathcal{M} \times \mathcal{M})$ of the "heat equation" with respect to the operator $A_{0}$

$$
\frac{\partial}{\partial t} K(t, x, y)+A_{0 x} K(s, x, y)=0
$$

with the initial condition in $C^{\infty}(\mathcal{M})$

$$
\lim _{t \rightarrow 0^{+}} K(t, x, y)=\delta(x, y) \text {. }
$$

Fixing a sufficiently small open geodesically convex neighborhood of the manifold $\mathcal{N} \mathfrak{F}$, the "heat-kernel expansion at $t \rightarrow 0^{+}$" is the decomposition of the heat kernel

$$
K(t ; x, y)=\frac{e^{-\sigma(x, y) / 2 t}}{(4 \pi t)^{D / 2}} \sum_{j=0}^{N} a_{j}(x, y) t^{j}+\frac{e^{-\eta \sigma(x, y) / 2 t}}{(4 \pi t)^{D / 2}} t^{N} O_{\eta, N}(t ; x, y),
$$

which holds for $x, y \in \mathcal{N}$. In (7), $\eta$ is a real which is arbitrarily fixed in $] 0,1[$ and the function $O_{\eta, N}$ satisfies

$$
\left|O_{\eta, N}(t ; x, y)\right|<C_{\eta, N}|t|
$$

uniformly in $(x, y)$. Above, $C_{\eta, N} \geq 0$ does not depend on $t$. Finally, the coefficients $a_{j}(x, y)$ are smooth functions defined in $\mathcal{N} \times \mathcal{N}$ by recurrence relations we shall examine shortly (see Mo98a and the appendix of Mo98b for details). Similar expansions have been studied extensively in physics and mathematics and have been generalized considering Laplace-like operators acting on smooth sections of vector bundles on Riemannian/Lorentzian manifolds (see Av98 and references therein). For $x \neq y$, the expansion above is not a proper asymptotic expansion because the remaining

$$
R_{N}(t ; x, y):=\frac{e^{-\eta \sigma(x, y) / 2 t}}{(4 \pi t)^{D / 2}} t^{N} O_{\eta, N}(t ; x, y),
$$

in principle, may be less infinitesimal than previous terms in the expansion in spite of vanishing faster than any positive power of $t$ as $t \rightarrow 0^{+}$. Taking the limit as $\eta \rightarrow 1^{-}$on the right-hand side of (9), one gets

$$
R_{N}(t ; x, y):=\frac{e^{-\sigma(x, y) / 2 t}}{(4 \pi t)^{D / 2}} t^{N} O_{N}(t ; x, y) .
$$

However, there is no guarantee that $O_{N}(t ; x, y)$ vanishes or is bounded as $t \rightarrow 0^{+}$. Therefore, as said above, the remaining of the formally "asymptotic" expansion of $K(t ; x, y)$ could be less infinitesimal than the previous terms in the expansion (7). The lack of general information of the precise behaviour of the remaining of the considered expansion

\footnotetext{
${ }^{3}$ Actually, with small changes, a very similar decomposition holds in the set $\mathcal{D}_{r}$ given above [Mo98a].
} 
around $t=0$ does not allow one to get important theorems such as the uniqueness of the coefficients $a_{j}(x, y)$. It is worthwhile stressing that, by the symmetry of $K(t ; x, y)$ in the Riemannian case and the general symmetry of $\sigma(x, y)$, the symmetry of the coefficients $a_{j}(x, y)$ would follow from the uniqueness theorem trivially. Actually, at least to the author's knowledge, there is no proof of the general off-diagonal asymptoticness of the heat-kernel expansion in the mathematical literaturef Conversely, there appear formulae concerning upper bounds of the heat kernel which contain some arbitrary parameter like $\eta$ above Da89. On the other hand, in practice, computations concerning off-diagonal heat-kernel coefficients in nontrivial cases are impossibly complicated and therefore, no counterexample is known concerning their symmetry. It is worthwhile remarking that symmetrized expansions for $K(t ; x, y)$ can be obtained following different approaches as the "Weyl calculus" [Ta96]. However, the coefficients obtained by this route satisfy different equations from the heat-kernel recurrence relations and, in general, cannot be identified with the standard heat-kernel coefficients used in physics. Obviously, in the case $x=y$, when both exponentials disappear, the heat-kernel expansion (7) is a proper asymptotic expansion.

Whenever $\mathcal{M}$ is Riemannian and noncompact, the heat kernel exists as a smooth function (see [Da89, Wa79 for the pure Laplacian case) and the expansion (7) remains true, in general, provided the injectivity radius of the manifold is strictly positive (this can be assured by imposing bounds on the sectional curvature of the manifold) and supposing that some bound conditions on the Ricci curvature tensor are satisfied [Ch84].

In the presence of boundaries of the Riemannian manifold $\mathcal{M}, A$ being some selfadjoint extension of $A_{0}$ determined by fixing some boundary conditions on $\partial \mathcal{M}$, the expansion above has to be changed just by adding a further (dependent on the boundary conditions) term $h(t ; x, y)$ in the sum above. However, for $x \neq y$, the literature on this case is not very extensive, except for the analysis of the pure Laplacian case with Dirichelet boundary conditions. In this case Ch84 $h(t ; x, y)$ can be bounded by a constant times $t^{D / 2} e^{-\sigma(y, \partial \mathcal{M}) / 4 t}$ ( or $x$ in place of $y$ ) and thus vanishes exponentially as $t \rightarrow 0^{+}$whenever at least one of the arguments does not belong to the boundary. In the case $x=y$, also $h(t ; x, x)$ can be expanded in an asymptotic series of terms. These terms carry powers of the form $t^{j+1 / 2-D / 2}$ instead of $t^{j-D / 2}$ ( $j$ natural) El95, EORBZ94 and maintain the exponential factor cited above. Hence, in the case $x=y$ away from the boundary, these added terms vanish faster than any power $t^{M}(M \in \mathbb{N})$ as $t \rightarrow 0^{+}$(see Ch84 for the pure Laplacian case).

In the Lorentzian case, the picture changes dramatically. Generally, $A_{0}$ is not bounded below and this drawback in general remains in self-adjoint extensions?. This introduces several pathologies dealing with the heat equation and the associated semigroup of expo-

\footnotetext{
${ }^{4}$ Unfortunately, the important textbook [Ch84] reports a result concerning this point which does not seem to follow from the corresponding proof (see Appendix of Mo98b ).

${ }^{5}$ They exist because $A_{0}$, thought as an operator on $L^{2}\left(\mathcal{M}, d \mu_{g}\right)$, is symmetric ( e.g., on $C_{0}^{\infty}(\mathcal{M})$ ) and it commutes with the antiunitary operator given by the complex conjugation [RS80.
} 
nentials which, as a consequence, contains unbounded operators. However, an analogous expansion should arise considering an "heat kernel" $H(s, x, y)$ solution of a "Schrödinger" equation [BD82, Fu91] formally related to the group of the imaginary exponentials operators (which are bounded) of the operator $A_{0}$,

$$
-i \frac{\partial}{\partial s} H(s, x, y)+A_{0 x} H(s, x, y)=0 ;
$$

with initial condition (holding on locally-integrable smooth test functions)

$$
\lim _{s \rightarrow 0} H(s, x, y)=\delta(x, y) .
$$

(See [Fu91, Ca90, Av98] for details.) In the Lorentzian case, one expects that some local "asymptotic" expansion of the form?

$$
H(t, x, y) \sim \frac{e^{i \sigma(x, y) / 2 s}}{(4 \pi i s)^{D / 2}} \sum_{j=0}^{\infty} a_{j}(x, y)(i s)^{j}
$$

should hold. If the manifold has a boundary, further terms appear and depend on the boundary. Actually, the situation is much more complicate [Fu91, EF97 and we shall not address this issue here. We only notice that, if the Lorentzian manifold is locally static and $V$ is invariant under the associated group of isometries, it should be possible to get information on the Lorentzian heat-kernel coefficients by a Wick-rotation into a Riemannian manifold. In this case, the analytical dependence of the heat-kernel coefficients on the time associated to the time-like Killing vector should be a consequence of the staticity of the metric. This should allow to perform the analytical continuation to the Euclidean time.

1.3 Determination and smoothness of heat-kernel and Hadamard coefficients. Let us consider a manifold $\mathcal{M}$ satisfying our general hypotheses. In a local coordinate system $x^{1}, x^{2}, \cdots, x^{D}$, defined in any open convex neighborhood or, more generally, in any open totally normal neighborhood $\mathcal{T}$, one can define the van Vleck-Morette determinant $\Delta_{V V M}$. This is a bi-scalar which is given in the coordinates above by Ca90

$$
\Delta_{V V M}(x, y):=-[g(x) g(y)]^{-1 / 2} \operatorname{det}\left(\frac{\partial^{2} \sigma(x, y)}{\partial x^{a} \partial y^{b}}\right)>0
$$

$x, y \in \mathcal{T}$. On $\mathcal{T}$, it satisfies (all derivatives are computed in the variable $x$ in the considered coordinate system)

$$
\nabla^{a} \sigma(x, y) \nabla_{a} \ln \Delta_{V V M}(x, y)=-D+\nabla_{c} \nabla^{c} \sigma(x, y) .
$$

\footnotetext{
${ }^{6}$ We stress that, if manifold is Lorentzian, $\sigma(x, y)$ can also be negative.
} 
Notice that $\Delta_{V V M}(x, y)$ is strictly positive on $\mathcal{T}$ (since it is a bi-scalar and $\Delta_{V V M}(x, y)=$ $|g(x)|^{-1 / 2}>0$ in normal coordinates around $\left.y\right)$ and it is a $C^{\infty}$ function of $(x, y)$ which is also analytic in $x$ and $y$ (separately in general) whenever the manifold and the metric are $C^{\infty}$ and analytic respectively. Obviously, this follows from the fact that $(x, y) \mapsto$ $\sigma(x, y)$ is $(x, y)-C^{\infty}$ or, respectively, analytic in $x$ and $y$ in the considered domain. If the manifold is Riemannian and the injectivity radius is strictly positive, the van VleckMorette determinant can be defined on the set $\mathcal{D}_{r}$ as a smooth function.

In either Riemannian or Lorentzian case, the coefficients $a_{j}$ are bi-scalars defined in any fixed open geodesically convex neighborhood $\mathcal{N}$ containing $x$ and $y$, away from the boundary of the manifold if it exists, or equivalently, in the set $\mathcal{D}_{r}$, provided the manifold is Riemannian with strictly positive injectivity radius. In the considered domain, the functions $(x, y) \mapsto a_{j}(x, y)$ can be heuristically determined by well-known equations with opportune regularity conditions. These equations are obtained perturbatively inserting the considered Lorentzian or Riemannian expansions formally computed up to $N=\infty$ (omitting the remaining) into (11) and (5) and imposing that each coefficient of any power of $t$ vanishes separately and taking into account (15). Following this route, in any normal coordinate system defined into a normal neighborhood of $y$, one finds

$$
\begin{aligned}
\frac{d}{d \lambda}\left(a_{0}(x(\lambda), y) \Delta_{V V M}^{-1 / 2}(x(\lambda), y)\right) & =0 \\
-\lambda^{j} \Delta_{V V M}^{-1 / 2}(x(\lambda), y) A_{0 x(\lambda)} a_{j}(x(\lambda), y) & =\frac{d}{d \lambda}\left(\lambda^{j+1} a_{j+1}(x(\lambda), y) \Delta_{V V M}^{-1 / 2}(x(\lambda), y)\right)
\end{aligned}
$$

where $\lambda \mapsto x(\lambda)$ is the unique segment geodesic from $y \equiv x(0)$ to $x \equiv x(1)$ completely contained in the normal neighborhood.

The regularity conditions are the following ones. The solutions have to be $(x, y)$-smooth everywhere in the considered domain, in particular they have to be bounded for $x \rightarrow y$. Moreover,

$$
a_{0}(x, y) \rightarrow 1
$$

must be hold for $x \rightarrow y$, which assures the validity of (12) and (6) since also $\Delta_{V V M}^{-1 / 2}(x, y) \rightarrow$ 1 .

The reason for using open geodesically convex neighborhoods should be clear. Indeed, in order to perform the derivatives contained in the differential operator $A_{0}$ on the left-hand side of (17), with $x$ and $y$ fixed in $\mathcal{N}$, there must exist an open neighborhood $\mathcal{O}_{z}$ of any point $z$ which belongs to the geodesic which connects $y$ with $x$, such that a geodesic which connect $y$ with any point in $\mathcal{O}_{z}$ lies completely in $\mathcal{N}$. Moreover the dependence of the considered geodesics on the extreme points has to be smooth. This is true provided $\mathcal{N}$ is open and geodesically convex, the smoothness being a consequence of the total normality of the neighborhood. (Working in $\mathcal{D}_{r}$ whenever possible, similar properties hold true and the definitions are well-posed.) 
The following definition gives the unique solutions in $\mathcal{N}$ of the recurrence equations (16) and (17) $(j \geq 1)$ satisfying the requirements given above, either for Riemannian and Lorentzian manifolds.

Definition 2.1. In our general hypotheses on $\mathcal{M}$ and $A_{0}$, where the former can admit a boundary and can be either Riemannian or Lorentzian, in any fixed open geodesically convex neighborhood $\mathcal{N}$ not intersecting $\partial \mathcal{M}$, the heat-kernel coefficients are the realvalued functions defined on $\mathcal{N} \times \mathcal{N}$, labeled by $j \in \mathbb{N}$,

$$
\begin{aligned}
a_{0}(x, y) & =\Delta_{V V M}^{1 / 2}(x, y) \\
a_{(j+1)}(x, y) & =-\Delta_{V V M}^{1 / 2}(x, y) \int_{0}^{1} \lambda^{j}\left[\Delta_{V V M}^{-1 / 2} A_{x(\lambda)} a_{j}\right](x(\lambda), y) d \lambda,
\end{aligned}
$$

$\lambda \mapsto x(\lambda)$ being the unique geodesic segment from $y \equiv x(0)$ to $x \equiv x(1)$ contained completely in $\mathcal{N}$.

(It is possible to give an analogous and equivalent definition on the set $\mathcal{D}_{r}$ in a Riemannian manifold with strictly positive injectivity radius. In any case, it is obvious that, fixing $x, y, a_{j}(x, y)$ defined above does not depend on the chosen open geodesically convex neighborhood containing $x$ and $y$ ).

In the case of a Riemannian compact manifold, the heat kernel coefficients defined above are just those which appear in (7) Ch84, Mo98a, Mo98b. Moreover, these coefficients do not depend on the particular self-adjoint extension of $A_{0}$. (Conversely, in the case of the presence of a boundary, the further coefficients cited previously do depend on the self-adjoint extension).

$a_{0}(x, y)$ enjoys the same properties of positivity, smoothness/analyticity of $\Delta_{V V M}(x, y)$. Moreover, assuming the smoothness/analyticity of the function $V$ which appears in the operator $A_{0}$ and working in local coordinates defined in a geodesically convex neighborhood containing $x$ and $y$, one can generalize this result to all the coefficients $a_{j}$. Indeed, taking account of the smooth/analytic dependence on the parameter and the initial and final conditions of the geodesics (and their derivatives) dC92, KN63 and finally considering (20), one can check that the coefficients $a_{j}(x, y)$ are $(x, y)$-smooth or $x$ and $y$ analytic functions of $(x, y)$ in the considered domain (away from the boundary). Concerning the proof of analyticity, a short way is to continue the functions on the right-hand side of (20) to complex values of the arguments $x$ and $y$. For example, since the integrand functions are analytic in $x$ for $y$ fixed, one can continue these to complex values in the variable $x$ for any fixed real $y$. Therefore one can prove Cauchy-Riemann's conditions for the complex components of $x$ on the left-hand side of (20) passing the derivatives under the sign of integration.

Also in the presence of boundaries, one can formally use the coefficients $a_{j}$ above (those which do not depend on the boundary conditions) to build up a part of a formal series 
for Green's functions $G(x, y)$

$$
A_{0 x} G(x, y)=\delta(x, y) \text {. }
$$

Indeed one has that the Green functions above can be locally approximated by formal series, which defines, whenever they converge to proper solutions, "Hadamard local fundamental solutions". Both in the Riemannian and in the Lorentzian case, these series can be represented, up to the indicated order, by (the summation appears for $D \geq 4$ only)

$$
\begin{aligned}
H(x, y)= & \sum_{j=0}^{D / 2-2}(D / 2-j-2) !\left(\frac{2}{\sigma}\right)^{D / 2-j-1} \frac{a_{j}(x, y \mid A)}{(4 \pi)^{D / 2}} \\
& -\frac{2 a_{D / 2-1}(x, y \mid A)-a_{D / 2}(x, y \mid A) \sigma}{2(4 \pi)^{D / 2}} \ln \left(\frac{\sigma}{2}\right)
\end{aligned}
$$

if $D$ is even, and (the summation appears for $D \geq 5$ only)

$$
\begin{aligned}
H(x, y)= & \sum_{j=0}^{(D-5) / 2} \frac{(D-2 j-4) ! ! \sqrt{\pi}}{2^{(D-3) / 2-j}}\left(\frac{2}{\sigma}\right)^{D / 2-j-1} \frac{a_{j}(x, y \mid A)}{(4 \pi)^{D / 2}} \\
& +\frac{a_{(D-3) / 2}(x, y \mid A)}{(4 \pi)^{D / 2}} \sqrt{\frac{2 \pi}{\sigma}}-\frac{a_{(D-1) / 2}(x, y \mid A)}{(4 \pi)^{D / 2}} \sqrt{2 \pi \sigma}
\end{aligned}
$$

if $D$ is odd, see Mo98b.

In fact, (17) and (19) assure that the coefficients of the formal series for the considered Green's function, truncated at the indicated orders, satisfy the corresponding recurrence differential equations given in Chapter 5 of Ga64 both in the Riemannian and in the Lorentzian case and the corresponding regularity/initial conditions. For $D$ even, the nondivergent part of the Hadamard expansion, omitted above, is ambiguous and depends on the choice of a coefficient. However, in the point-splitting technique, the symmetry is requested for the coefficients which appear above only. In the Lorentzian case one has to specify the prescription to compute the logarithms and the fractional powers of $\sigma$ in the case $\sigma<0$; as is well known in Quantum Field Theory, this produces differnt types of (Hadamard expansions of) two-point functions with the same coefficients (Wightman functions and Feynman propagator). The expansions above define "parametrices" of the Green's functions at the considered order of approximation. However, nothing assures that the corresponding not truncated series converge and, most important, define smooth functions. This convergence does anyway hold locally uniformly in the Riemannian case Ga64, in this case the series define proper functions. Furthermore, if one deals with a real section of a complex analytic manifold where the complex analytic function $V$ is also real, then the sum of the series is also analytic and thus smooth (by standard theorems of complex function theory) and define a true local solution of the corresponding differential equation: these are the Hadamard fundamental local solutions. The Lorentzian case, 
in particular cases at least, can be treated by analytic-continuation procedures from the Riemannian case, but, in general, one has a convergence in the sense of Borel only [Fu91, Fr75. However, all these issues should not be so important in practice, since, within the practical point-splitting procedure, one has to take into account only a finite number of terms of these expansions and thus one can use the parametrices instead of the sum of the series. Nevertheless the requirements above on the convergence of the series as well as the smoothness of the sum have been used within the proof of the conservation of the stress tensor [Wa78, Br84, BO86]. These requirements have been partially dropped in FSW78 where a "distributional" convergence of the Hadamard series have been used, but no explicit improved proof of the results given in Wa78 (and related papers) have been supplied.

As we said previously, another strongly important point, used to prove the conservation of the renormalized stress tensor in the cited literature, is the symmetry of the Hadamard local fundamental solutions concerning their divergent part as $\sigma \rightarrow 0$ Wa78 up to the order of expansion considered in (22) and (23) (actually the most part of the known literature treats the case $D=4$ only, but the same procedures can by generalized to different dimensions in a direct way). In [FSW78], it was argued that a proof of this property holds true also for the case of a $C^{\infty}$ (not analytic) manifold, unfortunately such a proof was not reported there and, at least to the author's knowledge, such a general proof (as well as a proof of the symmetry of the heat-kernel coefficients) does not exist in the literature. Notice that the symmetry of the heat-kernel coefficients assures the symmetry of the parametrices (22) and (23). For this reason the symmetry of the heatkernel coefficients is important in the point-splitting technique.

\section{A proof of the symmetry of heat kernel coefficients in the Riemannian case.}

2.1 Two theorems. Our proof is quite technical and involves several steps. The way is dealt with as follows. First, one shows that the thesis holds true in the case of a real analytic manifold by using known local properties of the expansion of the heat-kernel. This is the content of the first theorem we shall prove. Afterwards, one proves that, in some sense, any $C^{\infty}$ manifold can be approximated by analytic manifolds. This point is quite complicated because this approximation has to hold into a common geodesically convex neighborhood. This is necessary in order to make sensible a common definition of heat-kernel coefficients. Finally, one proves that the heat-kernel coefficients, defined in the common geodesically convex neighborhood are "sequentially continuous" in the class of the used metrics. Then, and this is the content of the second theorem we shall present, the symmetry for the case of a $C^{\infty}$ manifold follows by the "continuity" of the heat-kernel coefficients with respect to the metrics and from the symmetry in the analytic case. It is worthwhile stressing that (local and global) approximation theorems in real analytic 
manifolds are well-known in the literature (see TO98 for a recent review). However, these theorems concern functions rather than metrics and the problem of the existence of common geodesically convex neighborhoods is not treated explicitly. For this reason we prefer giving independent proofs (see the appendix of this work).

Lemma 2.1. Let us assume our general hypotheses on $A_{0}$ and $\mathcal{M}$ which is explicitly supposed to be Riemannian and compact. In a coordinate system defined in an open sufficiently small (geodesically convex) neighborhood $\mathcal{N}_{z}$ of any point $z \in \mathcal{M}$, for any pair of points $x, y \in \mathcal{N}_{z}$, and any natural $N$ such that $N>D / 2+2\left|\alpha^{\prime}\right|+2\left|\beta^{\prime}\right|, \alpha^{\prime}$, $\beta^{\prime}$ being arbitrarily fixed multi-indices, one has

$$
\begin{aligned}
D_{x}^{\alpha^{\prime}} D_{y}^{\beta^{\prime}} K(t ; x, y) & =D_{x}^{\alpha^{\prime}} D_{y}^{\beta^{\prime}}\left\{\frac{e^{-\sigma(x, y) / 2 t}}{(4 \pi t)^{D / 2}} \sum_{j=0}^{N} a_{j}(x, y) t^{j}\right\} \\
& +\frac{e^{-\eta \sigma(x, y) / 2 t}}{(4 \pi t)^{D / 2}} t^{N-\left|\alpha^{\prime}\right|-\left|\beta^{\prime}\right|} O_{\eta, N}^{\left(\alpha^{\prime}, \beta^{\prime}\right)}(t ; x, y) .
\end{aligned}
$$

Above, $\eta \in] 0,1\left[\right.$ can be fixed arbitrarily and the corresponding function $O_{\eta, N}^{\left(\alpha^{\prime}, \beta^{\prime}\right)}$ is continuous in $(t, x, y) \in\left[0,+\infty\left[\times \mathcal{N}_{z} \times \mathcal{N}_{z}\right.\right.$ and $(x, y)$-uniformly bounded by $B_{\eta, N}|t|$ in a positive neighborhood of $t=0, B_{\eta, N}>0$ being a constant.

Proof. See Lemma 2.1 of Mo98b.

By the lemma above we are able to prove our first result.

Theorem 2.1. In our general hypotheses on $\mathcal{M}$, which is supposed to be a Riemannian manifold (also with boundary in general), and $A_{0}$, the following properties hold true for the heat-kernel coefficients in (19) and (20).

(a) Fixed any point $z \in \mathcal{M}$ (away from $\partial \mathcal{M}$ ), there is a sufficiently small open geodesically convex neighborhood of $z, \mathcal{N}_{z}$ (which does not intersect $\partial \mathcal{M}$ ), such that, in any local coordinate system defined therein, for any $j \in \mathbb{N}$, and any pair of derivative operators $D_{x}^{\alpha}, D_{x}^{\beta}$ and for any point $y \in \mathcal{N}_{z}$

$$
\left.D_{x}^{\alpha} D_{y}^{\beta} a_{j}(x, y)\right|_{x=y}=\left.D_{x}^{\alpha} D_{y}^{\beta} a_{j}(y, x)\right|_{x=y} .
$$

(b) For any choice of the multi-indices $\alpha, \beta$ and $j, N \in \mathbb{N}$, the functions

$$
\Omega_{j}(x, y):=a_{j}(x, y)-a_{j}(y, x)
$$

computed in any local coordinate system in the set $\mathcal{N}_{z}$ defined in (a), satisfy

$$
\sigma^{-N}(x, y)\left[D_{x}^{\alpha} D_{y}^{\beta} \Omega_{j}(x, y)\right] \rightarrow 0
$$

as $x \rightarrow y$. 
(c) If $g_{a b}$ and $V$ are (real) analytic functions of some local coordinate frame defined in an open connected set $\mathcal{O}$ (away from the boundary) where, for all $x, y \in \mathcal{O}$, the coefficient $a_{j}(j$ fixed in $\mathbb{N})$ is defined, then

$$
a_{j}(x, y)=a_{j}(y, x)
$$

for any pair $(x, y) \in \mathcal{O} \times \mathcal{O}$.

Proof. Notice that the thesis is trivially proven for $a_{0}(x, y)=\Delta_{V V M}^{1 / 2}(x, y)$, since the righthand side is symmetric in $x$ and $y$. So we can pass directly to the case $j>0$ proving (a). Let us first consider the case of a compact Riemannian manifold. Then, since $A_{0}$ is positive, we can employ standard theorems on the heat kernel, in particular we can use Lemma 2.1 above. Therefore, let us fix a coordinate system where Lemma $\mathbf{2 . 1}$ holds true in an open geodesically convex neighborhood of the point $z$. For any pair of multi-indices $\alpha, \beta$,

$$
D_{x}^{\alpha} D_{y}^{\beta} \sum_{j=0}^{N} \Omega_{j}(x, y) t^{j}=(4 \pi t)^{D / 2} D_{x}^{\alpha} D_{y}^{\beta}\left\{e^{+\sigma(x, y) / 2 t}\left[\frac{e^{-\sigma(x, y) / 2 t}}{(4 \pi t)^{D / 2}} \sum_{j=0}^{N} \Omega_{j}(x, y) t^{j}\right]\right\} .
$$

Taking into account that $(x, y) \mapsto K(t ; x, y)-K(t ; y, x) \equiv 0$ in these hypotheses Ch84 (see also Theorem 1.1 in Mo98a ), employing Leibnitz' rule in evaluating the derivatives above and making use of (24), we get

$$
D_{x}^{\alpha} D_{y}^{\beta} \sum_{j=0}^{N} \Omega_{j}(x, y) t^{j}=t^{N-2|\alpha|-2|\beta|} e^{\delta \sigma(x, y) / 2 t} U_{\delta, N}^{(\alpha, \beta)}(t ; x, y),
$$

where $\delta=1-\eta \in] 0,1\left[\right.$ ( $\eta$ is the same parameter which appears in (24)), $U_{\delta, N}^{(\alpha, \beta)}(t ; x, y)$ is built up using linear combinations of antisymmetrized remainings which appear in (24), $O_{\eta, N}^{\left(\alpha^{\prime}, \beta^{\prime}\right)}(t ; x, y)-O_{\eta, N}^{\left(\alpha^{\prime}, \beta^{\prime}\right)}(t ; y, x)$ with coefficients given by positive powers of $t$ and derivatives of the function $\sigma$. Due to the similar property of the functions $O_{\eta, N}^{\left(\alpha^{\prime}, \beta^{\prime}\right)}$, $(t ; x, y) \mapsto U_{\delta, N}^{(\alpha, \beta)}$ is $(x, y)$-uniformly bounded by some constant $C_{\delta, N}>0$ in a rightneighborhood of $t=0$, provided $N$ has been chosen large sufficiently. Then, taking the limit for $x \rightarrow y$ we have

$$
\left.\sum_{j=0}^{N} D_{x}^{\alpha} D_{y}^{\beta} \Omega_{j}(x, y)\right|_{x=y} t^{j}=t^{N-2|\alpha|-2|\beta|} U_{\delta, N}^{(\alpha, \beta)}(t ; y, y),
$$

and thus, with a trivial redefinition of $U$ obtained by decomposing

$$
\sum_{j=0}^{N}=\sum_{j=0}^{N-2|\alpha|-2|\beta|-1}+\sum_{N-2|\alpha|-2|\beta|}^{N}
$$


one gets

$$
\left.\sum_{j=0}^{N-2|\alpha|-2|\beta|-1} D_{x}^{\alpha} D_{y}^{\beta} \Omega_{j}(x, y)\right|_{x=y} t^{j}=t^{N-2|\alpha|-2|\beta|} V_{\delta, N}^{(\alpha, \beta)}(t ; y, y),
$$

where $V_{\delta, N}^{(\alpha, \beta)}(t ; y, y)$ is bounded in a positive neighborhood of $t=0$. In the limit $t \rightarrow 0^{+}$, this is possible only when all the coefficients of the polynomial on the left-hand side vanish separately. This implies that also the covariant derivatives of any order of the functions $\Omega_{j}$, evaluated on the diagonal, vanish. Obviously this does not depend on the particular coordinate frame used around $y$. Therefore, changing coordinates and passing from covariant derivatives to ordinary derivatives in a different coordinate frame, we get that, once again, the derivatives of any order of the functions $\Omega_{j}$, evaluated on the diagonal vanish. (a) has been proven in the hypotheses of a compact manifold (without boundary). Given a general manifold $\mathcal{M}$ and any inner point $y$, we can consider a neighborhood $\mathcal{O}$ of $y$ and build up a new manifold $\mathcal{M}^{\prime}$ which contains a neighborhood $\mathcal{O}^{\prime}$ isometric to $\mathcal{O}$. $\mathcal{M}^{\prime}$ can be chosen compact (without boundary) provided $\mathcal{M}$ is complete. On $\mathcal{M}^{\prime}$, we can define an operator $A_{0}^{\prime}$ (depending on a smooth potential $V^{\prime}$ ) which coincides with $A_{0}$ in the neighborhood $\mathcal{O}^{\prime} \equiv \mathcal{O}$. In general, also if $A_{0}$ is positive, $A_{0}^{\prime}$ may be non-positive. However, since $V^{\prime}$ is bounded below by some real $v$, the operator $A_{0}^{\prime}+|v| I$ is positive on $\mathcal{M}^{\prime}$. We can consider the heat-kernel coefficients $b_{j}(x, y)$ of the expansion (7) for the operator $A+|v| I$. For these coefficients the item (a) of the thesis holds true. An algebraic computation based on the fact that, formally, if $S(t ; x, y)$ satisfies the heat equation with respect to $A_{0}$ then $S(t ; x, y) \exp (-c t)$ satisfies the same equation with respect to $A_{0}+c I$ $(c \in \mathbb{R})$, proves that the coefficients $a_{j}(x, y)$ of (19) and (20) corresponding to $A_{0}^{\prime} \equiv A_{0}$ are related to those above by the relations

$$
\begin{aligned}
& a_{j}(x, y)=\sum_{k=0}^{j} \frac{(-1)^{k}|v|^{k} b_{j-k}(x, y)}{k !}, \\
& b_{j}(x, y)=\sum_{k=0}^{j} \frac{|v|^{k} a_{j-k}(x, y)}{k !} .
\end{aligned}
$$

The coefficients on the right-hand side of (32) satisfy (19) and (20) with respect to $A_{0} \equiv A_{0}^{\prime}$ in $\mathcal{O} \equiv \mathcal{O}^{\prime}$ once the coefficients on the right-hand side do so with respect to $A_{0}^{\prime}-|v| I$. Thus, the item (a) is trivially proven for the coefficients $a_{j}(x, y)$ in the general case. Notice that, in the same way, also items (b) and (c) hold true in the general case provided they are valid in the particular case of a compact manifold without boundary.

The item (b) is trivially proven by expanding, in the variable $x$, any $\Omega_{j}(x, y)$ and all of its derivatives, via Taylor algorithm, around the point $y$ in a normal Riemannian coordinate system centered in $y \equiv 0$. For instance, considering $\Omega_{j}$, one has, for any $N \in \mathbb{N}$

$$
\Omega_{j}(x, y)=\left.\sum_{0 \leq|\alpha| \leq 2 N+1} \frac{\left(x^{1}\right)^{\alpha_{1}} \cdots\left(x^{D}\right)^{\alpha_{D}}}{\alpha_{1} ! \cdots \alpha_{D} !} \frac{\partial^{|\alpha|} \Omega(x, 0)}{\partial^{\alpha_{1}} x^{1} \cdots \partial^{\alpha_{D}} x^{D}}\right|_{x=0}+|x|^{2 N+1} O_{2 N+1}(x) \text {, }
$$


where $|x|^{2} / 2=\sigma(x, y)$, and $O_{2 N+1}(x)$ is a smooth function which vanishes as $x \rightarrow 0 \equiv y$ and thus is bounded around $y \equiv 0$. Using the result of the item (a) (changing coordinates in general), one gets the thesis (b). The same procedure can be employed for derivatives of $\Omega_{j}(x, y)$.

Let us consider the item (c). In this case, the Taylor expansion above can be carried out, in the considered coordinates, up to $N=\infty$. Thus, taking into account (a), for $x$ belonging to a neighborhood of any fixed point $y \in \mathcal{O}$, one has

$$
\Omega_{j}(x, y)=0 \text {. }
$$

Since, for $y$ fixed in $\mathcal{O}, \Omega_{j}(x, y)$ is analytic in $x \in \mathcal{O}$, which is an open and connected set, and vanishes in an open neighborhood contained in $\mathcal{O}$ (dependent on $y$ ), it has to vanish everywhere on $\mathcal{O}$. Therefore $(x, y) \mapsto \Omega_{j}(x, y)$ vanishes in $\mathcal{O} \times \mathcal{O}$.

The results obtained above concerning the heat-kernel coefficients, can be generalized directly to the relevant coefficients $u_{j}, v_{j}$ of the expansions of the Hadamard local solution of the operator $A_{0}$ by taking into account (22) and (23) above. Actually, the result contained in the item (b) should be sufficient for all applications of (Euclidean) point-splitting procedures known in the literature despite the complete symmetry of the Hadamard coefficients was originally requested[. However, we aim to get a more general result.

Proposition 2.1. Let $\mathcal{M}$ be a real $C^{\infty}$ manifold with a non-singular metric $\mathbf{g}$, satisfying our general hypotheses.

(a) Let $\Omega^{\prime}$ be any open set in $\mathcal{M}$ (such that $\Omega^{\prime} \cap \partial \mathcal{M}=\emptyset$ ) endowed with a coordinate frame $x^{1}, \cdots, x^{D}$. For any connected relatively-compact open set $\Omega$, such that $\bar{\Omega} \subset \Omega^{\prime}$, there is a sequence of real metrics $\left\{\mathbf{g}_{n}\right\}$ with the same signature of $\mathbf{g}$ defined in a neighborhood of $\bar{\Omega}$ such that each $g_{n a b}$ is an analytic function of the given coordinates and the sequence $\left\{\mathbf{g}_{n}\right\}$ converges uniformly in $\bar{\Omega}$ to the metric $\mathbf{g}$. Similarly, for any fixed multiindex $\alpha$, the sequance of derivatives with respect to the coordinates $x^{1}, \cdots, x^{D}\left\{D^{\alpha} \mathbf{g}_{n}\right\}$, converges uniformly in $\bar{\Omega}$ to $\left\{D^{\alpha} \mathbf{g}\right\}$.

(b) For any choice of the set $\Omega^{\prime}$, the coordinates $x^{1}, \cdots, x^{D}$, the set $\Omega$ and the sequence $\left\{\mathbf{g}_{n}\right\}$ given above and for any $z \in \Omega$, there is a natural $N_{0}$ and a family of open neighborhoods of $z,\left\{\mathcal{N}_{z i}\right\}, i \in \mathbb{R}$, such that $\left\{\mathcal{N}_{z i}\right\}$ is a local base of the topology of $\mathcal{M}$, $\mathcal{N}_{z i} \subset \overline{\mathcal{N}}_{z i^{\prime}} \subset \Omega$, for any pair $i, i^{\prime}$ such that $i^{\prime}>i$ and, moreover, for any $i \in \mathbb{R}$, both $\mathcal{N}_{z i}$ and $\overline{\mathcal{N}}_{z i}$ are common geodesically convex neighborhoods of $z$ for all the metrics $\mathbf{g}$ and $\mathbf{g}_{n}$ when $n>N_{0}$.

(c) For any choice of the set $\Omega^{\prime}$, the coordinates $x^{1}, \cdots, x^{D}$, the set $\Omega$, the sequence $\left\{\mathbf{g}_{n}\right\}, z \in \Omega$ and the class $\left\{\mathcal{N}_{z i}\right\}, i \in \mathbb{R}$ arbitrary, the functions $(x, y) \mapsto \sigma_{n}(x, y)$ are welldefined and smooth in any neighborhood of $\overline{\mathcal{N}}_{z i} \times \overline{\mathcal{N}}_{z i}$ and the sequence of these functions

\footnotetext{
${ }^{7}$ I am grateful to R. M. Wald for this remark.
} 
as well as the sequences of their derivatives of any order converge uniformly in $\overline{\mathcal{N}}_{z i} \times \overline{\mathcal{N}}_{z i}$ to $\sigma(x, y)$ and corresponding derivatives.

(d) For any choice of the set $\Omega^{\prime}$, the coordinates $x^{1}, \cdots, x^{D}$, the set $\Omega$, the sequence $\left\{\mathbf{g}_{n}\right\}, z \in \Omega$ and the class $\left\{\mathcal{N}_{z i}\right\}$, for any $i \in \mathbb{R}$, if $(\lambda, x, y) \mapsto \gamma_{n}(\lambda, x, y), \lambda \in[0,1]$, indicates the only geodesic segments starting from the point $y \in \mathcal{N}_{z i}$ and terminating in the point $x \in \overline{\mathcal{N}}_{z i}$ corresponding to the $n$-th metric and contained in $\overline{\mathcal{N}}_{z i}$, then $\left\{\gamma_{n}(\lambda, x, y)\right\}$ and the sequences of their $\lambda, x, y$-derivatives of any order converge uniformly in $[0,1] \times \overline{\mathcal{N}}_{z i} \times \overline{\mathcal{N}}_{z i}$ to $\gamma(\lambda, x, y)$ and corresponding derivatives, $\gamma(\lambda, x, y)$ being the geodesic of the initial metric $\mathbf{g}$.

\section{Proof. See Appendix $\square$.}

We need another technical lemma to get the final theorem.

Lemma 2.2. Let $\left\{g_{k, n}\right\}$ be a class of continuous functions, $k=1,2, \cdots, l$ and $n \in$ $\mathbb{N} \cup\{\infty\}$,

$$
g_{k, n}: K_{k} \rightarrow M_{k},
$$

where $M_{k}$ and $K_{k} \subset M_{k}$ are, respectively, metric spaces and compact sets. Let $\left\{f_{n}\right\}$ be a class of continuous functions, $n \in \mathbb{N} \cup\{\infty\}$,

$$
f_{n}: \Omega_{1} \times \Omega_{2} \times \cdots \times \Omega_{l} \rightarrow N,
$$

where $N$ is a metric space, the sets $\Omega_{k} \subset M_{k}, k=1,2, \cdots, l$, are open and $g_{k, \infty}\left(K_{k}\right) \subset \Omega_{k}$. Suppose that, for any fixed $k$ and for $n \rightarrow+\infty, g_{k, n} \rightarrow g_{k, \infty}$ uniformly in $K_{k}$ and $f_{n} \rightarrow f_{\infty}$ uniformly in $\Omega_{1} \times \cdots \times \Omega_{l}$. Then, there is a natural $N_{0}$ such that, for $n>N_{0}$, the left-hand side below is well-defined and, for $n \rightarrow+\infty$,

$$
f_{n}\left(g_{1, n}\left(x_{1}\right), g_{2, n}\left(x_{2}\right), \cdots, g_{l, n}\left(x_{l}\right)\right) \rightarrow f_{\infty}\left(g_{1, \infty}\left(x_{1}\right), g_{2, \infty}\left(x_{2}\right), \cdots, g_{l, \infty}\left(x_{l}\right)\right)
$$

uniformly in $K_{1} \times K_{2} \times \cdots \times K_{l}$.

Proof. It is quite straightforward. Take into account that a continuous function $h$ defined on a compact set $H$ of a metric space with values in a metric space is uniformly continuous in $H$ and $h(H)$ is also a compact set. $N_{0}$ is defined by determining the compact sets $C_{1}, \cdots, C_{l}$ such that $g_{k}\left(K_{k, n}\right) \subset C_{k} \subset \Omega_{k}$, for $n=N_{0}+1, N_{0}+2, \cdots, \infty$.

We are now able to state and prove the most important theorem concerning the symmetry of heat-kernel coefficients in Riemannian manifold.

Theorem 2.2. Let $M$ be a $C^{\infty}$ Riemannian manifold (with boundary in general) and $A_{0}$ an operator, both satisfying our general hypotheses. For any point $z \in \mathcal{M}$ (away from 
$\partial \mathcal{M})$ there is a geodesically convex neighborhood of $z, \mathcal{N}_{z}$ (which does not intersect $\partial \mathcal{M}$ ) such that, for any pair $(x, y) \in \mathcal{N}_{z}$

$$
a_{j}(x, y)=a_{j}(y, x)
$$

for $j=0,1,2 \cdots$, where the heat-kernel coefficients are those given in (19) and (20).

Proof. Fix any $z \in \mathcal{M}$ away from the boundary, let $\Omega^{\prime}$ be an open set endowed with a coordinate frame $x^{1}, \cdots, x^{D}\left(\Omega^{\prime} \cap \partial \mathcal{M}=\emptyset\right)$ and let $\Omega$ be a connected relatively-compact open neighborhood of $z$ such that $\bar{\Omega} \subset \Omega^{\prime}$. We can use the thesis of Proposition 2.1 with the same notations employed there. In particular, fix a common open geodesically convex neighborhood $\mathcal{N}_{z}:=\mathcal{N}_{z i_{0}}$ of $z$ and its closure given in Proposition 2.1 and a sequence of analytic metrics $\left\{\mathbf{g}_{n}\right\}$ defined in a neighborhood of the compact set $\bar{\Omega}$ also given in Proposition 2.1. Since $\mathcal{N}_{z}$ and $\overline{\mathcal{N}}_{z}$ are geodesically convex and, by Proposition 2.1, there is another similar open neighborhood $\mathcal{N}_{z i}$, such that $\overline{\mathcal{N}}_{z} \subset \mathcal{N}_{z i}$, both coefficients $a_{j}(x, y)$ and $a_{j}(y, x)$ are well-defined and smooth in $\overline{\mathcal{N}}_{z} \times \overline{\mathcal{N}}_{z}$. For the moment, let us suppose also that $V$ is an analytic function of the considered coordinates. Let us fix $x, y \in \mathcal{N}_{z}$, and consider the functionals of the metrics defined in $\overline{\mathcal{N}}_{z}$

$$
\begin{aligned}
& a_{j x y}\left[\mathbf{g}_{n}\right]:=(-1)^{j} \Delta^{-1 / 2}\left(x, y \mid \mathbf{g}_{n}\right) a_{j}\left(x, y \mid \mathbf{g}_{n}\right), \\
& a_{j y x}\left[\mathbf{g}_{n}\right]:=(-1)^{j} \Delta^{-1 / 2}\left(y, x \mid \mathbf{g}_{n}\right) a_{j}\left(y, x \mid \mathbf{g}_{n}\right),
\end{aligned}
$$

$n=0,1, \cdots \infty$, with $\mathbf{g}_{\infty}:=\mathbf{g}$ and $\Delta:=\Delta_{V V M}$. If $\mathbf{g}_{n}$ is fixed, the coefficients above are smooth functions on $\overline{\mathcal{N}}_{z} \times \overline{\mathcal{N}}_{z}$ which are also analytic in $x$ and $y$. Obviously, the symmetry of these functionals in $x, y$ would involve that of the heat-kernel coefficients since the VVM determinant is symmetric. Since $\overline{\mathcal{N}}_{z}$ is totally normal, it is possible to make explicit each $a_{j x y}$ and $a_{j x y}$ in terms of a sequence of integrals computed along the unique geodesic between $x$ and $y$ which belongs completely to a normal neighborhood centered on $x$ (as well as $y$ ) including the whole set $\overline{\mathcal{N}}_{z}$. Moreover, since $\overline{\mathcal{N}}_{z}$ is geodesically convex with respect to all the metrics, we can do it for all the metrics $\mathbf{g}_{n}$, the corresponding geodesics depending on the particular metric one is considering. Let us indicate the considered geodesic starting from $y$ and reaching $y^{\prime} \in \overline{\mathcal{N}}_{z}$ and computed with respect to the metric $\mathbf{g}_{n}$ by $\lambda \mapsto \gamma\left(\lambda, y^{\prime}, y \mid \mathbf{g}_{n}\right)$ (with $\left.\lambda \in[0,1]\right)$. Employing (19) and (20), one finds, with $A_{0 x}[\mathbf{g}]=-\nabla_{\mathbf{g} a} \nabla_{\mathbf{g}}^{a}+V$

$$
\begin{aligned}
a_{0 x y}[\mathbf{g}] \equiv & 1, \\
a_{1 x y}[\mathbf{g}]= & \int_{0}^{1} d \lambda\left[\Delta^{-1 / 2} A_{0}\right]_{\gamma(\lambda, x, y)}[\mathbf{g}] \Delta^{1 / 2}(\gamma(\lambda, x, y \mid \mathbf{g}), y \mid \mathbf{g}), \\
a_{2 x y}[\mathbf{g}]= & \int_{0}^{1} d \lambda \int_{0}^{1} d \lambda^{\prime} \lambda^{\prime}\left[\Delta^{-1 / 2} A_{0}\right]_{\gamma\left(\lambda^{\prime}, x, y\right)}[\mathbf{g}] \Delta^{1 / 2}\left(\gamma\left(\lambda^{\prime}, x, y \mid \mathbf{g}\right), y \mid \mathbf{g}\right) \\
& \times\left[\Delta^{-1 / 2} A_{0}\right]_{\gamma\left(\lambda, \gamma\left(\lambda^{\prime}, x, y\right), y\right)}[\mathbf{g}] \Delta^{1 / 2}\left(\gamma\left(\lambda, \gamma\left(\lambda^{\prime}, x, y \mid \mathbf{g}\right), y \mid \mathbf{g}\right), y \mid \mathbf{g}\right), \\
& \cdots
\end{aligned}
$$




$$
a_{j x y}[\mathbf{g}]=\int_{0}^{1} d \lambda \int_{0}^{1} d \lambda_{1} \lambda_{1}^{1} \cdots \int_{0}^{1} d \lambda_{j-1} \lambda_{j-1}^{j-1} \mathcal{A}_{j x y}\left(\lambda, \lambda_{1}, \cdots \lambda_{j-1} \mid \mathbf{g}\right)
$$

where (omitting the explicit dependence on the chosen metric for sake of simplicity)

$$
\begin{aligned}
\mathcal{A}_{j x y}\left(\lambda, \lambda_{1}, \cdots \lambda_{j-1}\right):= \\
\\
{\left[\Delta^{-1 / 2} A_{0}\right]_{\gamma\left(\lambda_{j-1}, x, y\right)} \Delta^{1 / 2}\left(\gamma\left(\lambda_{j-1}, x, y\right), y\right) \times } \\
{\left[\Delta^{-1 / 2} A_{0}\right]_{\gamma\left(\lambda_{j-2}, \gamma\left(\lambda_{j-1}, x, y\right), y\right)} \Delta^{1 / 2}\left(\gamma\left(\lambda_{j-2}, \gamma\left(\lambda_{j-1}, x, y\right), y\right), y\right) \times } \\
\quad \cdots \\
{\left[\Delta^{-1 / 2} A_{0}\right]_{\gamma\left(\lambda, \gamma\left(\lambda_{1}, \cdots \gamma\left(\lambda_{j-1}, x, y\right), \cdots y\right), y\right)} \Delta^{1 / 2}\left(\gamma\left(\lambda, \gamma\left(\lambda_{1}, \cdots \gamma\left(\lambda_{j-1}, x, y\right), \cdots y\right), y\right), y\right) }
\end{aligned}
$$

Notice that, fixing any metric, $\Delta(x, y)$ and their derivatives are smooth functions of the derivatives of the function $\sigma(x, y)$ in the set $\overline{\mathcal{N}}_{z} \times \overline{\mathcal{N}}_{z}$, therefore by item (c) of Proposition 2.1 and Lemma 2.2, on the compact set $\overline{\mathcal{N}}_{z} \times \overline{\mathcal{N}}_{z}$, one gets the uniform convergence with all of the derivatives of the sequence of functions $\Delta^{ \pm 1 / 2}\left(x, y \mid \mathbf{g}_{n}\right)$ to the function $\Delta^{ \pm 1 / 2}(x, y \mid \mathbf{g})$. Moreover, from item (d) of Proposition 2.1, taking into account that all the functions appearing in the integration above are computed on the geodesics connecting $y$ with $x$ which, not depending on $n$, belong completely to the compact $\overline{\mathcal{N}}_{z}$, and using recurrently Lemma 2.2 one gets

(1) for $j=0,1,2, \cdots$

$$
\mathcal{A}_{j x y}\left(\lambda, \lambda_{1}, \cdots \lambda_{j-1} \mid \mathbf{g}_{n}\right) \rightarrow \mathcal{A}_{j x y}\left(\lambda, \lambda_{1}, \cdots \lambda_{j-1} \mid \mathbf{g}\right)
$$

as $n \rightarrow+\infty$. This holds uniformly in $\lambda, \lambda_{1}, \cdots, \lambda_{j-1} \in[0,1]$

and therefore,

(2) for any $j \in \mathbb{N}$, there is a constant $C_{j}$ such that

$$
\left|\mathcal{A}_{j x y}\left(\lambda, \lambda_{1}, \cdots \lambda_{j-1} \mid \mathbf{g}\right)\right|<C_{j} \quad \text { for } n=1,2, \cdots,
$$

uniformly in $\left(\lambda, \lambda_{1}, \cdots, \lambda_{j-1}\right) \in[0,1]^{j}$. Lebesgue's dominated convergence theorem assures that, for $n \rightarrow+\infty$,

$$
a_{j x y}\left[\mathbf{g}_{n}\right] \rightarrow a_{j x y}[\mathbf{g}] .
$$

The same result can be obtained considering the coefficients $a_{j y x}\left[\mathbf{g}_{n}\right]$ and $a_{j y x}[\mathbf{g}]$. This allows one to conclude the proof noticing that,

$$
\left(a_{j x y}\left[\mathbf{g}_{n}\right]-a_{j y x}\left[\mathbf{g}_{n}\right]\right) \rightarrow\left(a_{j x y}[\mathbf{g}]-a_{j y x}[\mathbf{g}]\right)
$$

for $n \rightarrow+\infty$. The left-hand side above vanishes because the metrics $\mathbf{g}_{n}$ are analytic in the open connected set $\mathcal{N}_{z} \times \mathcal{N}_{z}$ and thus the item (c) of Theorem 2.2 holds true. If $V=V(x)$ is not analytic, one can find a sequence of positive analytic functions of the considered coordinates in $\overline{\mathcal{N}}_{y},\left\{V_{n}\right\}$, such that this sequence converges uniformly to 
$V$ with all of its derivatives. This sequence can be obtained considering the convolutions of $V$ and the flat-space heat kernel similarly to what we have done in building up the sequence of the metrics $\mathbf{g}_{n}$ for proving Proposition 2.1 (see Appendix). Defining $A_{0 x}\left[\mathbf{g}_{n}\right]:=-\nabla_{\mathbf{g}_{n}}^{a} \nabla_{\mathbf{g}_{n}}^{a}+V_{n}$, and using the same arguments above, one can prove (44) and (45) once again and therefore gets the thesis.

We have a straightforward corollary based on the formulae (22) and (23).

Corollary of Theorem 2.2. Let $M$ be a D-dimensional $C^{\infty}$ Riemannian manifold and $A_{0}$ an operator both satisfying our general hypotheses. For any point $z \in \mathcal{M}$ (away from $\partial \mathcal{M}$ if $\partial \mathcal{M}$ is not empty) there is a geodesically convex neighborhood $\mathcal{N}_{y}$ of $y$, (which does not intersect $\partial \mathcal{M}$ if $\partial \mathcal{M}$ exists) such that, for any pair $(x, y) \in \mathcal{N}_{z}$, up to the orders indicates in the summations below, the coefficients $u_{j}, v_{j}$ of the Hadamard parametrix

$$
H(x, y)=\sum_{j=0}^{D / 2-2}\left(\frac{2}{\sigma(x, y)}\right)^{D / 2-j-1} u_{j}(x, y)+\sum_{j=0}^{2} v_{j}(x, y) \ln (\sigma(x, y) / 2)
$$

for $D$ even (the summation appears for $D \geq 4$ only), and

$$
\begin{aligned}
H(x, y)= & \sum_{j=0}^{(D-5) / 2}\left(\frac{2}{\sigma(x, y)}\right)^{D / 2-j-1} u_{j}(x, y)+v(x, y) \sqrt{\frac{2 \pi}{\sigma(x, y)}} \\
& +w_{0}(x, y) \sqrt{2 \pi \sigma(x, y)}
\end{aligned}
$$

for $D$ odd (the summation appears for $D \geq 5$ only), satisfy

$$
\begin{aligned}
& u_{j}(x, y)=u_{j}(y, x), \\
& v_{j}(x, y)=v_{j}(y, x) .
\end{aligned}
$$

2.2 Conclusions and Outlooks. We have proven the symmetry of the heat-kernel/Hadamard coefficients in the general Riemannian case. The Lorentzian case remains an open issue. However, we expect that one can pass to the Lorentzian case from the Riemannian one by some analytic continuation, if the manifold and the coefficient $V$ are analytic. This should assure the symmetry of the considered coefficients in the analytic Lorentzian case. From that, the symmetry in the $C^{\infty}$ Lorentzian case is straightforward, since the proof of Theorem 2.2 needs the validity of the symmetry in the analytic case only, not depending on the signature of the metric. Indeed, Proposition 2.1, which is the kernel of the proof above, holds true for any signature of the metric of the manifold (and some parts of it can be generalized for non-metric affine connections). 


\section{Appendix: Proof of Proposition 2.1.}

Several simple lemmata are necessary. We do not report the proofs of those lemmata for the sake of brevity. These are based essentially on the Banach fixed-point theorem, the theorem of the inverse function and further simple considerations of elementary real analysis?

Lemma A1. Let $f$ be a function of $C^{k}\left(\left[t_{0}-\Delta, t_{0}+\Delta\right] \times \bar{B}_{R}\left(y_{0}\right) ; \mathbb{R}^{m}\right)$, where $k \in\{\infty, \omega\}$, $t_{0}, \Delta>0$ and $R>0$ are real numbers and $B_{R}\left(y_{0}\right)$ indicates the open ball of $\mathbb{R}^{m}$ centered in $y_{0}$ with radius $R$. Let us consider the differential equation

$$
\frac{d Y}{d t}=f(t, Y) \quad Y \in C^{k}\left(\left[t_{0}-\delta, t_{0}+\delta\right] ; \mathbb{R}^{m}\right) \quad \text { for some } \quad \delta>0, \delta \leq \Delta
$$

with initial condition

$$
Y\left(t_{0}\right)=\bar{y}_{0} \quad \bar{y}_{0} \in \bar{B}_{r}\left(y_{0}\right), r \text { fixed arbitrarily such that } 0<r<R .
$$

(a) A solution of Eq. (52) with initial condition (53) exists and is unique in any set $\left[t_{0}-\delta, t_{0}+\delta\right]$, provided that

$$
0<\delta<\operatorname{Min}\left(\Delta, \Delta_{r}^{\prime}, \Delta^{\prime \prime}\right)
$$

where $\Delta_{r}^{\prime}=[(R-r) / 2] /\left\{\operatorname{Sup}\left\{\|f(t, y)\| \mid t \in\left[t_{0}-\Delta, t_{0}+\Delta\right] y \in \bar{B}_{R}\left(y_{0}\right)\right\}\right\}$ and

$\Delta^{\prime \prime}=1 /\left\{\operatorname{Sup}\left\{\sqrt{\operatorname{Tr} \nabla f(t, y)^{T} \nabla f(t, y)} \mid t \in\left[t_{0}-\Delta, t_{0}+\Delta\right] y \in \bar{B}_{R}\left(y_{0}\right)\right\}\right\}$.

(b) It satisfies $Y\left(t, \bar{y}_{0}\right) \in \bar{B}_{R}\left(y_{0}\right)$ for any $t \in\left[t_{0}-\delta, t_{0}+\delta\right]$ and $\bar{y}_{0} \in \bar{B}_{r}\left(y_{0}\right)$.

(c) Moreover, the solution $\left(t, \bar{y}_{0}\right) \mapsto Y\left(t, \bar{y}_{0}\right)$ belongs to $C^{\infty}\left(\left[t_{0}-\delta, t_{0}+\delta\right] \times \bar{B}_{r}\left(y_{0}\right) ; \mathbb{R}^{m}\right)$ and, in the case $k=\omega$, it is also analytic in the variable $t \in\left[t_{0}-\delta, t_{0}+\delta\right]$ and in the variable $\bar{y}_{0} \in \bar{B}_{r}\left(y_{0}\right)$ (separately in general).

Lemma A2. Let $\left\{f_{n}\right\}$ be a sequence of functions of $C^{\infty}\left(\left[t_{0}-\Delta, t_{0}+\Delta\right] \times \bar{B}_{R}\left(y_{0}\right) ; \mathbb{R}^{m}\right)$, where the used notations are the same as those used in the Lemma A1. Let us suppose also that, for any $p=0,1,2 \cdots$ and for any multi-index $\alpha$,

$$
D_{y}^{\alpha} \frac{\partial^{p} f_{n}}{\partial t^{p}} \rightarrow D_{y}^{\alpha} \frac{\partial^{p} f_{\infty}}{\partial t^{p}} \quad \text { uniformly on }\left[t_{0}-\Delta, t_{0}+\Delta\right] \times \bar{B}_{R}\left(y_{0}\right),
$$

$f_{\infty}$ being another function of $C^{\infty}\left(\left[t_{0}-\Delta, t_{0}+\Delta\right] \times \bar{B}_{R}\left(y_{0}\right) ; \mathbb{R}^{m}\right)$.

Let us indicate the solutions of the equation (52), with $f_{n}$ in place of $f$ and initial condition (53), by $\left(t, \bar{y}_{0}\right) \mapsto Y_{n}\left(t, \bar{y}_{0}\right)(n=0,1,2, \cdots, \infty)$. Then, for any $\delta>0$ satisfying (54) above with $f_{\infty}$ in place of $f$, and any $r>0$ with $r<R$ :

\footnotetext{
${ }^{8} \mathrm{~A}$ complete proof of the Lemmata contained in this appendix can be found within the first version of the preprint gr-qc/9902034 http://xxx.lanl.gov/abs/gr-qc/9902034v1).
} 
(a) There is a natural $N_{\epsilon}$ such that for $n>N_{\epsilon},\left(t, \bar{y}_{0}\right) \mapsto Y_{n}\left(t, \bar{y}_{0}\right)$ is defined in $\left[t_{0}-\delta, t_{0}+\right.$ $\delta] \times \bar{B}_{r}\left(y_{0}\right)$;

(b) for any $p=0,1,2, \cdots$,

$$
\frac{\partial^{p} Y_{n}}{\partial t^{p}} \rightarrow \frac{\partial^{p} Y_{\infty}}{\partial t^{p}} \quad \text { uniformly in }\left[t_{0}-\delta, t_{0}+\delta\right] \times \bar{B}_{r}\left(y_{0}\right)
$$

Lemma A3. With the same hypotheses of Lemma A2 one gets also that, for any $p=0,1,2, \cdots$ and for any multi-index $\alpha$,

$$
D_{\bar{y}_{0}}^{\alpha} \frac{\partial^{p} Y_{n}}{\partial t^{p}} \rightarrow D_{\bar{y}_{0}}^{\alpha} \frac{\partial^{p} Y_{\infty}}{\partial t^{p}} \quad \text { uniformly in }\left[t_{0}-\delta, t_{0}+\delta\right] \times \bar{B}_{r}\left(y_{0}\right)
$$

Lemma A4. Let $\left\{f_{n}\right\}$ be a sequence of $\mathbb{R}^{m}$-valued functions defined in an open set $N \subset \mathbb{R}^{m}$ such that:

(i) $f_{n} \in C^{k}\left(N ; \mathbb{R}^{m}\right)$ for $n=0,1, \cdots$, where $k$ is fixed in $\{\infty, \omega\}$.

(ii) $f_{n} \rightarrow f_{\infty} \in C^{k}\left(N ; \mathbb{R}^{m}\right)$ uniformly in the set $N$ with all of their derivatives of any order.

(iii) There is a point $x_{0} \in N$ such that $\operatorname{det}\left(\left.\nabla f_{\infty}\right|_{x=x_{0}}\right) \neq 0$.

Then, there exist two open neighborhoods of $x_{0}$ and $f_{\infty}\left(x_{0}\right)$ respectively, $\mathcal{U}_{x_{0}}$ and $\mathcal{V}_{f\left(x_{0}\right)}$, a real $K>0$ and a natural $N_{0}$ such that, for $n>N_{0}$ including $n=\infty$,

(a) all functions $\left.f_{n}\right|_{\mathcal{U}_{x_{0}}}: \mathcal{U}_{x_{0}} \rightarrow f_{n}\left(\mathcal{U}_{x_{0}}\right)$ define diffeomorphisms, in particular, any $f_{n}\left(\mathcal{U}_{x_{0}}\right)$ is an open set. Moreover, $\left|\operatorname{det}\left(\nabla f_{n} \mid \mathcal{U}_{x_{0}}\right)\right|>K$.

(b)

$$
\mathcal{V}_{f_{\infty}\left(x_{0}\right)} \subset \bigcap_{n>N_{0}} f_{n}\left(\mathcal{U}_{x_{0}}\right)
$$

where the intersection includes $n=\infty$.

(c) In the set $\mathcal{V}_{f_{\infty}\left(x_{0}\right)}$ and for $n \rightarrow \infty, f_{n}^{-1} \rightarrow f_{\infty}^{-1}$ uniformly with all of their derivatives of any order. Moreover, $0<\left|\operatorname{det} \nabla\left(\left.f_{n}^{-1}\right|_{\mathcal{V}_{f_{\infty}\left(x_{0}\right)}}\right)\right|<\frac{1}{K}$.

Lemma A5. Let $K$ be a connected compact set of $\mathbb{R}^{m}$ and $G, G_{n}: K \rightarrow M(D, \mathbb{R})$ continuous functions such that $G(x)=G(x)^{T}$ and $G_{n}(x)=G_{n}(x)^{T}$ for any $x \in K$, $n=0,1,2, \cdots ; M(D, \mathbb{R})$ denoting the algebra of real $D \times D$ matrices. Let us suppose that $G(x)$ is not singular for any $x \in K$ and, component by component, $G_{n}(x) \rightarrow G(x)$ uniformly in $x$, for $n \rightarrow \infty$. Then, there is a natural $N_{0}$ such that, for $n>N_{0}$ and not depending on $x \in K$, all the matrices $G_{n}(x)$ are non-singular and $\operatorname{sign}\left(G_{n}(x)\right)=\operatorname{sign}(G(x))$, $\operatorname{sign}(A)$ denoting the signature of the real symmetric matrix $A$. 


\section{Proof of Proposition 2.1.}

Let us proceed with the proof of the item (a). Let $\Omega^{\prime}$ be an open neighborhood of the point $z$ in the manifold $\mathcal{M}$, such that $\Omega^{\prime} \cap \partial \mathcal{M}=\emptyset$. Suppose also that $\vec{x} \equiv\left(x^{1}, \cdots, x^{D}\right)$ are coordinates defined in $\Omega^{\prime}$. Then, let $\Omega$ be a connected relatively-compact open neighborhood of $z$ such that $\bar{\Omega} \subset \Omega^{\prime}$.

Let $\mathbf{g}$ be the metric on $\mathcal{M}$ which can be either Lorentzian or Riemannian. Finally, let us define the pure Euclidean-Laplacian heat kernel in $\mathbb{R}^{D}$,

$$
E(t, \vec{x}, \vec{y}):=\frac{e^{-|\vec{x}-\vec{y}|^{2} / 4 t}}{(4 \pi t)^{D / 2}},
$$

where $\vec{x}, \vec{y} \in \mathbb{R}^{D}$ and $\left.t \in\right] 0,+\infty[$. From now on, we shall identify the various subsets of $\Omega^{\prime}$ with the corresponding subsets of $\mathbb{R}^{D}$ through the given coordinate system. Since the topology on $\Omega^{\prime}$ is that of $\mathbb{R}^{D}$, one can find another connected relatively-compact open set $\Omega^{\prime \prime}$ such that $\bar{\Omega} \subset \Omega^{\prime \prime}$ and $\bar{\Omega}^{\prime \prime} \subset \Omega^{\prime}$. Let us consider the class of covariant second-order tensorial fields defined in the given coordinate system on $\Omega^{\prime}$,

$$
g_{n a b}(\vec{x}):=\int_{\mathbb{R}^{D}} d^{D} \vec{y} E(1 / n, \vec{x}, \vec{y}) g_{a b}(\vec{y}) \eta(\vec{y}),
$$

where $d^{D} \vec{y}$ is the natural Lebesgue measure on $\mathbb{R}^{D}$ and $\vec{x} \mapsto \eta(\vec{x})$ is a nonnegative $C^{\infty}$ function which takes the value 1 in $\bar{\Omega}$ and vanishes outside of $\bar{\Omega}^{\prime \prime}$. From the wellknown properties of the Euclidean heat-kernel Ch84 we have that, since $y \mapsto g_{a b}(\vec{y}) \eta(\vec{y})$ in (60) is uniformly continuous in its domain (as it is continuous in a compact set), $g_{n a b}(\vec{x}) \rightarrow g_{a b}(\vec{x}) \eta(\vec{x})$ uniformly in $\mathbb{R}^{D}$, as $n \rightarrow \infty$. In particular, this holds in $\bar{\Omega}$ where $\eta(\vec{x})=1$. Therefore, for any point $\vec{x} \in \bar{\Omega}$, we have a sequence of symmetric matrices $G_{n}(\vec{x}) \equiv\left[g_{n a b}(\vec{x})\right]$ which converges to the nonsingular symmetric matrix $G(\vec{x}) \equiv\left[g_{a b}(\vec{x})\right]$ uniformly in $\vec{x} \in \bar{\Omega}$. By Lemma A5, for $n>N_{0}$, the matrices $G_{n}(\vec{x})$ define metrics in the tangent space at $\vec{x}$ with the same signature of $G(\vec{x})$.

The uniform convergence in $\bar{\Omega}$ given above holds also for the derivatives of any order of the components $g_{n a b}(\vec{x})$ and $g_{a b}(\vec{x})$. Indeed, from (60), one has, passing the derivatives under the sign of integration (see the extended discussion below) and then, using the integration by parts,

$$
\begin{aligned}
D_{\vec{x}}^{\alpha} g_{n a b}(\vec{x}) & =\int_{\mathbb{R}^{D}} d^{D} \vec{y} D_{\vec{x}}^{\alpha} E(1 / n, \vec{x}, \vec{y}) g_{a b}(\vec{y}) \eta(\vec{y}) \\
& =\int_{\mathbb{R}^{D}} d^{D} \vec{y}(-1)^{|\alpha|}\left(D_{\vec{y}}^{\alpha} E(1 / n, \vec{x}, \vec{y})\right) g_{a b}(\vec{y}) \eta(\vec{y}) \\
& =\int_{\mathbb{R}^{D}} d^{D} \vec{y} E(1 / n, \vec{x}, \vec{y}) D_{\vec{y}}^{\alpha}\left(g_{a b}(\vec{y})\right) \eta(\vec{y})+G^{(\alpha)}(\vec{x}) .
\end{aligned}
$$

The function $G^{(\alpha)}$ above is a sum of terms containing derivatives of order $>0$ of the function $\eta$; omitting overall constants, these terms are of the form $(|\gamma|>0)$

$$
\int_{\mathbb{R}^{D}} d^{D} \vec{y} E(1 / n, \vec{x}, \vec{y}) D_{\vec{y}}^{\beta}\left(g_{a b}(\vec{y})\right) D_{\vec{y}}^{\gamma} \eta(\vec{y})
$$


Taking $\vec{x} \in \bar{\Omega}$ and $n \rightarrow \infty$ these terms converge to the functions $D_{\vec{x}}^{\beta}\left(g_{a b}(\vec{x})\right) D_{\vec{x}}^{\gamma} \eta(\vec{x})$ which vanish in $\bar{\Omega}$ since $\eta$ is constant in $\bar{\Omega}$ and $|\gamma|>0$. Therefore, dropping the term $G^{(\alpha)}$, one has from (61), for $\vec{x} \in \bar{\Omega}$ and $n \rightarrow \infty$,

$$
D_{\vec{x}}^{\alpha} g_{n a b}(\vec{x}) \rightarrow D_{\vec{x}}^{\alpha} g_{a b}(\vec{x}),
$$

uniformly in $\vec{x}$.

To conclude the proof of the item (a), let us prove that, fixing the indices $a, b$, the functions $\vec{x} \mapsto g_{a b}(\vec{x})$ are analytic functions of the coordinates $\vec{x}$ on the whole space $\mathbb{R}^{D}$.

From (60) and the definition of the function $\eta$, we have

$$
g_{n a b}(\vec{x}):=\int_{\bar{\Omega}^{\prime \prime}} d^{D} \vec{y} E(1 / n, \vec{x}, \vec{y}) g_{a b}(\vec{y}) \eta(\vec{y}) .
$$

Fixing the natural $n$ and $\vec{y} \in \bar{\Omega}^{\prime \prime}$, it is possible to continue the variable $\vec{x}$ of the heatkernel to complex values. It is obvious that, fixing $n=1,2, \cdots$, the obtained function $(\vec{\zeta}, \vec{y}) \mapsto E(1 / n, \vec{\zeta}, \vec{y})$ belongs to $C^{\infty}\left(\mathbb{C}^{D} \times \mathbb{R}^{D}\right)$ and, for any fixed $\vec{y}$, it is holomorphic in the variable $\vec{\zeta} \in \mathbb{C}$. Obviously, the derivatives of any order in $\operatorname{Re} \vec{\zeta}$ and $\operatorname{Im} \vec{\zeta}$ of the integrand of (60) are bounded in any compact set of the form $\overline{\mathcal{O}}_{\vec{\zeta}_{0}} \times \bar{\Omega}^{\prime \prime}, \mathcal{O}_{\vec{\zeta}_{0}}$ being a relatively compact open neighborhood of $\vec{\zeta}_{0} \in \mathbb{C}^{D}$. Therefore, Lebesgue's dominated convergence theorem implies that the left hand side of (64) continued to complex values of $\vec{x}=\vec{\zeta}$ is smooth and one can pass the derivatives in (any component of) $\operatorname{Re} \vec{\zeta}$ and $\operatorname{Im} \vec{\zeta}$ under the sign of integration. In this way one can check the validity of Cauchy-Riemann's conditions for the $\vec{y}$-integrated function of $\vec{\zeta}$ by the validity of the same conditions for the integrand function of $(\vec{\zeta}, \vec{y})$. Therefore $\vec{\zeta} \mapsto g_{a b}(\vec{\zeta})$ is a complex-analytic function. Taking $\operatorname{Im} \vec{\zeta}=0$, one gets the real-analyticity of the left-hand side of (64).

Let us pass to prove the item (b). Our strategy is the following: We shall define the exponential maps of each metric $\mathbf{g}_{n}$ around the point $z \equiv \overrightarrow{0}$ and thus, by using these exponential maps and by shrinking the found neighborhoods, we shall define normal neighborhoods, totally normal neighborhoods and geodesically convex neighborhoods of the metrics $\mathbf{g}_{n}$. Finally, we shall extract a class of (totally normal) geodesically convex neighborhoods which are common to all metrics.

Let us fix $z \in \Omega$, define a normal coordinate system $\vec{y}$ with respect to the metric $\mathbf{g}_{\infty}:=\mathbf{g}$ and centered in $z \equiv \overrightarrow{0}$. This coordinate system is defined in a normal neighborhood centered in $z, \mathcal{N}_{z}=B_{\eta}(\overrightarrow{0}), \eta>0$, the open ball above being defined in the normal coordinates with respect to the standard $\mathbb{R}^{D}$ metric (obviously, this defines open sets with respect to the topology of the manifold). Employing Lemma 2.2, one sees that, in this system of coordinates one still has $g_{n a b}(\vec{y}) \rightarrow g_{\infty a b}(\vec{y})$ uniformly in $\vec{y}$, with all the derivatives. However, in general, the components of the various metrics are not analytic functions of the coordinates, but this is not important at this step.

In the considered coordinates, the first order geodesical equations read, for any metric $\mathbf{g}_{n}$ 
(including $n=\infty$ ),

$$
\begin{aligned}
& \frac{d y_{n}^{a}\left(t, \vec{y}_{0}, \vec{v}_{0}\right)}{d t}=v_{n}^{a}\left(t, \vec{y}_{0}, \vec{v}_{0}\right) \\
& \frac{d v_{n}^{a}\left(t, \vec{y}_{0}, \vec{v}_{0}\right)}{d t}=-\Gamma_{n b c}^{a}\left(\vec{y}_{n}\right) v^{b}\left(t, \vec{y}_{0}, \vec{v}_{0}\right) v^{c}\left(t, \vec{y}_{0}, \vec{v}_{0}\right) .
\end{aligned}
$$

(The sum over the repeated indices is understood). Above, $\vec{y}_{0}$ and $\vec{v}_{0}$ are, respectively, the initial position and the initial velocity evaluated at $t=0$. The latter, in components, defines a vector in $T_{\vec{y}}(\mathcal{M})$. From Lemma $\mathbf{A} 1$, we know that the solution is unique provided that $\left(t,\left(\vec{y}_{0}, \vec{v}_{0}\right)\right) \in[-\delta, \delta] \times \bar{B}_{R}((\overrightarrow{0}, \overrightarrow{0}))$ for some $\delta>0$ and $R>0$. We can find a real $r>0$ such that $\bar{B}_{r}(\overrightarrow{0}) \times \bar{B}_{r}(\overrightarrow{0}) \subset \bar{B}_{R}((\overrightarrow{0}, \overrightarrow{0}))$ (in any case, $\bar{B}_{r}(\overrightarrow{0}) \subset \Omega$ must hold). Obviously, the existence and uniqueness of the solution holds true also replacing $\bar{B}_{R}((\overrightarrow{0}, \overrightarrow{0}))$ by $\bar{B}_{r}(\overrightarrow{0}) \times \bar{B}_{r}(\overrightarrow{0})$. Let us indicate the geodesics given above in coordinates by $\gamma_{n}$. From Lemma A2 and Lemma A3, we know that, in the considered common domain, employing coordinates $\vec{y}$ and for $n$ larger than some $N_{0}, \gamma_{n}\left(t, \vec{y}_{0}, \vec{v}_{0}\right) \rightarrow \gamma_{\infty}\left(t, \vec{y}_{0}, \vec{v}_{0}\right)$ with all the $t, \vec{y}_{0}, \vec{v}_{0}$ derivatives, uniformly in all these variables, where $\left(t, \vec{y}_{0}, \vec{v}_{0}\right) \mapsto \gamma_{\infty}\left(t, \vec{y}_{0}, \vec{v}_{0}\right)$ is the geodesic associated to the target metric $\mathbf{g}_{\infty}=\mathbf{g}$.

For any fixed real $\alpha>0,(65)$ and (66) entail the identity

$$
\gamma_{n}\left(\alpha t, \vec{y}_{0}, \vec{v}_{0} / \alpha\right)=\gamma_{n}\left(t, \vec{y}_{0}, \vec{v}_{0}\right)
$$

for $n=0,1, \cdots, \infty$. This means that, if $2>\delta>0$, maintaining all properties concerning the uniform convergence and passing to the new variable $\lambda=(2 / \delta) t$, we can work with geodesics defined in the interval $\lambda \in[-2,2]$ provided $r$ is replaced by $r^{\prime}=(\delta / 2) r<r$. Since there is no ambiguity we can use the name $r$ instead of $r^{\prime}$. Therefore, from now on, we suppose $\lambda \in[-2,2]$. This allows one to define the well-known exponential maps for $(\vec{y}, \vec{v}) \in \bar{B}_{r}(\overrightarrow{0}) \times \bar{B}_{r}(\overrightarrow{0})$,

$$
(\vec{y}, \vec{v}) \mapsto \exp _{n y}(\vec{v}):=\gamma_{n}(1, \vec{y}, \vec{v}) .
$$

Once the exponential maps are defined in the common neighborhood above, we can pass to study the totally normal neighborhoods.

To this end, let us consider the functions, defined in our coordinate system and in the induced base in the tangent space,

$$
F_{n}: \bar{B}_{r}(\overrightarrow{0}) \times \bar{B}_{r}(\overrightarrow{0}) \rightarrow \mathcal{M} \times \mathcal{M}:(\vec{y}, \vec{v}) \mapsto\left(\vec{y}, \exp _{n y}(\vec{v})\right) .
$$

Notice that, in the considered domain, for $n \rightarrow \infty$, the sequence of $F_{n}(\vec{y}, \vec{v})$, like the sequences of their derivatives of any order in $(\vec{x}, \vec{v})$, converge to $F_{\infty}(\vec{y}, \vec{v})$ and corresponding derivatives of it, uniformly in these variables.

Passing from the geodesic equations (65), (66) to the corresponding equations for the $\vec{y}, \vec{v}$ derivatives of the solutions, one can straightforwardly prove that $\operatorname{det}\left(\left.\nabla F_{\infty}\right|_{(\vec{y}, \vec{v})=(\overrightarrow{0}, \overrightarrow{0})}\right)=1$. 
(Obviously, this property holds true for any $n$ and any point $\vec{y}$ in the considered domain.) Therefore, using Lemma A4 in the set $B_{r}(\overrightarrow{0}) \times B_{r}(\overrightarrow{0})$, one gets that there is a common neighborhood of $(\overrightarrow{0}, \overrightarrow{0}), \mathcal{U}_{(\overrightarrow{0}, \overrightarrow{0})} \subset B_{r}(\overrightarrow{0}) \times B_{r}(\overrightarrow{0})$ where all functions $F_{n}$, for $n>N_{0}^{\prime}$, define diffeomorphisms. Moreover there is an open neighborhood of $F_{\infty}((\overrightarrow{0}, \overrightarrow{0}))=(\overrightarrow{0}, \overrightarrow{0}), \mathcal{V}_{(\overrightarrow{0}, \overrightarrow{0})}$ such that

$$
\mathcal{V}_{(\overrightarrow{0}, \overrightarrow{0})} \subset \bigcap_{n>N_{0}^{\prime}} F_{n}\left(\mathcal{U}_{(\overrightarrow{0}, \overrightarrow{0})}\right)
$$

Without loss of generality, we can take $\mathcal{U}_{(\overrightarrow{0}, \overrightarrow{0})}$ of the form $\mathcal{U}_{\overrightarrow{0}} \times B_{\rho}(\overrightarrow{0}), 0<\rho<r, \overline{\mathcal{U}}_{\overrightarrow{0}}$ being an open neighborhood of $\overrightarrow{0}$. Similarly, we can take $\mathcal{V}_{(\overrightarrow{0}, \overrightarrow{0})}$ of the form $\mathcal{V}_{\overrightarrow{0}} \times \mathcal{V}_{\overrightarrow{0}}$.

In any open neighborhood of $(\overrightarrow{0}, \overrightarrow{0}), \mathcal{V}_{(\overrightarrow{0}, \overrightarrow{0})}$ satisfying $(\overrightarrow{70})$, the inverse of the functions $F_{n}$ converges uniformly with all the derivatives to the inverse of $F_{\infty}$, and $F_{n}^{-1}$ and $F_{\infty}^{-1}$ are diffeomorphisms. Therefore as $n \rightarrow \infty, \exp _{n \vec{y}}^{-1}\left(\vec{y}^{\prime}\right) \rightarrow \exp _{\infty \vec{y}}^{-1}\left(\vec{y}^{\prime}\right)$ uniformly in $\left(\vec{y}, \vec{y}^{\prime}\right) \in$ $\mathcal{V}_{\overrightarrow{0}} \times \mathcal{V}_{\overrightarrow{0}}$

This enable us to prove that $\mathcal{V}_{\overrightarrow{0}}$ is a totally normal neighborhood of $z \equiv \overrightarrow{0}$ for all the considered metrics. Take $\vec{y} \in \mathcal{V}_{\overrightarrow{0}}$. From the definition of $F_{n}$ and (70), one has

$$
\{\vec{y}\} \times \mathcal{V}_{\overrightarrow{0}} \subset F_{n}\left(\{\vec{y}\} \times B_{\rho}(\overrightarrow{0})\right),
$$

and therefore, $\vec{v} \mapsto \exp _{n \vec{y}}(\vec{v})$ is a diffeomorphism in $B_{\rho}(\overrightarrow{0})$ and for any $n>N_{0}$ including $n=\infty$,

$$
\mathcal{V}_{\overrightarrow{0}} \subset \exp _{n \vec{y}}\left(B_{\rho}(\overrightarrow{0})\right) \text { for any } \vec{y} \in \mathcal{V}_{\overrightarrow{0}}
$$

This means that $\mathcal{V}_{\overrightarrow{0}}$ is a totally normal neighborhood of $z \equiv \overrightarrow{0}$ which is common for all metrics provided $n>N_{0}^{\prime}$.

In this last step, we prove that it is possible to choose $\mathcal{V}_{\overrightarrow{0}}$ such that $\mathcal{V}_{\overrightarrow{0}}$ and $\overline{\mathcal{V}}_{\overrightarrow{0}}$ are common geodesically convex neighborhoods of $z \equiv \overrightarrow{0}$ for all the metrics whenever $n>N_{0} \geq N_{0}^{\prime}$. Actually, we shall find a class of neighborhoods $\mathcal{V}_{\overrightarrow{0}}$ defining a local base of the topology. Essentially we shall use the theory developed in the part 8 of Chapter III of [KN63.

The set $\mathcal{V}_{\overrightarrow{0}}$ can be chosen as a ball $B_{\delta}(\overrightarrow{0})$.

Our thesis can be proven using the following two results:

[1] There is an integer $N_{0}^{\prime \prime}>N_{0}^{\prime}$ and a real $\rho^{\prime}>0$, such that for $n>N_{0}^{\prime \prime}$ (including $n=\infty)$, the $D \times D$-matrix-valued functions, given in components by

$$
A_{n a b}(\vec{y}):=\left(\delta_{a b}-\sum_{c} \Gamma_{n a b}^{c}(\vec{y}) y^{c}\right)
$$

are positive definite for $\vec{y} \in B_{\rho^{\prime}}(\overrightarrow{0})$.

Above, the connection coefficients $\Gamma_{n a b}^{c}$ are those corresponding to the $n$-th metric represented in coordinates $\vec{y}$. 
[2] Fixed an open ball $B_{\rho^{\prime}}(\overrightarrow{0})$ with $\rho^{\prime}>0$ sufficiently small, it is possible to choose a natural $N_{0}>N_{0}^{\prime \prime}$, two reals $\rho>0$ and $\delta^{\prime}>0$ such that, for $n>N_{0}$ (including $n=\infty$ ), using coordinates $\vec{y}$ in the domain as well as in the co-domain,

$$
\exp _{n \vec{y}}\left(B_{\rho}(\overrightarrow{0})\right) \subset B_{\rho^{\prime}}(\overrightarrow{0}) \quad \text { for any } \vec{y} \in B_{\delta^{\prime}}(\overrightarrow{0}) .
$$

Before we prove [1] and [2], we show that [1] and [2] entail that for any real $\delta$ such that $0<\delta<\delta^{\prime}$, one has $\mathcal{N}_{z}:=\mathcal{V}_{\overrightarrow{0}}:=B_{\delta}(\overrightarrow{0})$ and its closure is geodesically convex with respect to all metrics. The item (b) is therefore completely proved by posing, in the considered coordinates, $\mathcal{N}_{z i}:=B_{\delta}(\overrightarrow{0})$ with $i=-\cot \left(\pi \delta / \delta^{\prime}\right)$. Notice that the open neighborhoods of $z$ given above define a local base of the topology because one can define a Riemannian metric in a neighborhood of $z$ such that the balls $B_{\delta}(\overrightarrow{0})$ are metric balls. Moreover, it is well known KN63 that the metric topology induced from any metric on a manifold coincides with the topology of the manifold.

To this end, in our coordinate frame, let us indicate a geodesic of the $n$-th metric by $\lambda \mapsto \vec{y}_{n}(\lambda)$ and consider the function $\lambda \mapsto T_{n}(\lambda)=\sum_{a}\left(y^{a}(\lambda)\right)^{2}$. Suppose that such a geodesic is tangent to the boundary of a ball $\partial B_{\epsilon}(\overrightarrow{0})(\epsilon>0)$ for $\lambda=0$. From the geodesic equations, one gets

$$
\left.\frac{d^{2} T_{n}}{d \lambda^{2}}\right|_{\lambda=0}=2 \sum_{a, b}\left[\left.\left.\left(\delta_{a b}-\sum_{c} \Gamma_{n a b}^{c}\left(\vec{y}_{n}(0)\right) y_{n}^{c}(0)\right) \frac{d^{2} y_{n}^{a}}{d \lambda^{2}}\right|_{\lambda=0} \frac{d^{2} y_{n}^{b}}{d \lambda^{2}}\right|_{\lambda=0}\right] \text {. }
$$

Therefore [1] assures that, if $\epsilon<\rho^{\prime}$, there is a neighborhood of the tangent point where the geodesics lie outside $B_{\epsilon}(\overrightarrow{0})$ for $n>N_{0}^{\prime \prime}$ (including $n=\infty$ ).

Now we use [2] to conclude the proof. Let us choose $\rho$ in (71) and $\delta^{\prime}>0$, such that (73) is satisfied, $\rho^{\prime}$ being that considered in [1]. We want to show that $\mathcal{V}_{\overrightarrow{0}}=B_{\delta}(\overrightarrow{0})$ and $\overline{\mathcal{V}}_{\overrightarrow{0}}$ are geodesically convex for any metrics $\mathbf{g}_{n}, n>N_{0}$ (including $n=\infty$ ) if $0<\delta<\delta^{\prime}$. Let $\vec{y}_{1}$ and $\vec{y}_{2}$ be any couple of points of $B_{\delta}(\overrightarrow{0})$ (or $\bar{B}_{\delta}(\overrightarrow{0})$ ). Consider the $n$-geodesic $t \mapsto \vec{y}_{n}(\lambda), \lambda \in[0,1]$, joining these points. We want to show that it lies in $B_{\delta}(\overrightarrow{0})\left(\bar{B}_{\delta}(\overrightarrow{0})\right.$ respectively). Suppose that this is not true. Then, there is at least one point which does not belong to $B_{\delta}(\overrightarrow{0})\left(\bar{B}_{\delta}(\overrightarrow{0})\right.$ respectively). In our hypotheses, the maximum of the function $T_{n}$ is attained for a value of the parameter $\left.\lambda_{n e} \in\right] 0,1$ [ since the extreme points of the geodesics belong to $B_{\delta}(\overrightarrow{0})\left(\bar{B}_{\delta}(\overrightarrow{0})\right.$ respectively) and thus $T_{n}(0), T_{n}(1)<T_{n}\left(\lambda_{n e}\right)$. Therefore, posing $\vec{y}_{n e}:=\vec{y}_{n}\left(\lambda_{n e}\right)$ and $\rho_{n e}:=T_{n}\left(\lambda_{n e}\right)$, since $\lambda_{n e}$ is an internal point of $[0,1], d T_{n} /\left.d \lambda\right|_{\lambda=\lambda_{n e}}=0$ must hold and thus the geodesic is tangent to $\partial B_{\rho_{n e}}(\overrightarrow{0})$ at $\vec{y}_{n e}$ where $T_{n}$ reaches its maximum. Notice that, because of [2], all the geodesics lie in $B_{\rho^{\prime}}(\overrightarrow{0})$. Therefore, due to [1], there is a neighborhood of $\vec{y}_{n e}=\vec{y}_{n}\left(\lambda_{n e}\right)$ where the geodesics lie outside $B_{\rho_{n e}}(\overrightarrow{0})$. This is not possible. This means that, not depending on $n>N_{0}, B_{\delta}(\overrightarrow{0})$ and $\bar{B}_{\delta}(\overrightarrow{0})$ are geodesically convex. (Actually, the maximum of $T_{n}$ is attained at the extreme points where the geodesic is not tangent to the corresponding sphere, and thus there is no absurdum.) 
To conclude the proof of the item (b), let us prove [1] and [2] above.

The proof of [1] is quite simple. As we know, one has that, for a sufficiently small ball centered in $\overrightarrow{0}$, the metrics $\mathbf{g}_{n}$, represented in coordinates $\vec{y}$, converge uniformly to the metric $\mathbf{g}_{\infty}$ with all the derivatives. Therefore, in a sufficiently small ball centered in $\overrightarrow{0}, \Gamma_{n a b}^{c}(\vec{y})$ must converge uniformly in $\vec{y}$ to $\Gamma_{\infty a b}^{c}(\vec{y})$ for $n \rightarrow \infty$, and thus, from (72), $\left\|A_{n}-A_{\infty}\right\|_{\infty} \rightarrow 0$. Then notice that $\Gamma_{\infty a b}^{c}(\overrightarrow{0})=0$, because the coordinates $\vec{y}$ are normal coordinates of the metric $\mathbf{g}_{\infty}$ centered on $\overrightarrow{0}$. Therefore, there is a sufficiently small ball centered in $\overrightarrow{0}$ where $A_{\infty}(\vec{y})$ is positive definite uniformly in $\vec{y}$, i.e., there exists an $a>0$ such that $\left(u, A_{\infty}(\vec{y}) u\right)>a$ uniformly in $\vec{y}$ and $u$ with $\|u\|=1$. In fact, since $\|u\|=1$,

$$
\left|\left(u,\left(A_{\infty}(\vec{y})-A_{\infty}(\overrightarrow{0})\right) u\right)\right| \leq\left\|A_{\infty}(\vec{y})-A_{\infty}(\overrightarrow{0})\right\|,
$$

and $\left\|A_{\infty}(\vec{y})-A_{\infty}(\overrightarrow{0})\right\| \rightarrow 0$ as $\vec{y} \rightarrow \overrightarrow{0}$. Since the bound above is uniform in $u$, one has that, for any $\epsilon>0$, there is a neighborhood of $\vec{y}=\overrightarrow{0}$ where,

$$
\left(u, A_{\infty}(\vec{y}) u\right)>\left(u, A_{\infty}(\overrightarrow{0}) u\right)-\epsilon=1-\epsilon,
$$

uniformly in $u$. Taking $\epsilon>0$ such that $1-\epsilon=a>0, a$ is the requested positive lower bound of $\left(u, A_{\infty}(\vec{y}) u\right)$. Now, we can repeat the same procedure considering the norm \|\|$_{\infty}$ and different values of $n$ in the found neighborhood. One has

$$
\left|\left(u,\left(A_{n}(\vec{y})-A_{\infty}(\vec{y})\right) u\right)\right| \leq\left\|A_{\infty}-A_{n}\right\|_{\infty} .
$$

Since $\left\|A_{n}-A_{\infty}\right\|_{\infty} \rightarrow 0$ as $n \rightarrow 0$, we get that, for any $\epsilon>0$, there is a $N_{0}^{\prime \prime}$ such that, for $n>N_{0}^{\prime \prime}$,

$$
\left(u, A_{n}(\vec{y}) u\right)>\left(u, A_{\infty}(\vec{y}) u\right)-\epsilon>a-\epsilon,
$$

uniformly in $u$ and $\vec{y}$. Taking $0<\epsilon<a$, one has proventhe thesis.

Let us prove [2]. The case $n=\infty$ is trivial since the function $(\vec{y}, \vec{v}) \mapsto \exp _{\infty \vec{y}}(\vec{v})$ is continuous and $\exp _{\infty \overrightarrow{0}}(\overrightarrow{0})=\overrightarrow{0}$. We also know that, for sufficiently small $\rho, \delta>0$, in $B_{\delta^{\prime}}(\overrightarrow{0}) \times B_{\rho}(\overrightarrow{0})$, the sequence of functions $(\vec{y}, \vec{v}) \mapsto \exp _{n \vec{y}}(\vec{v})$ converges to the function $(\vec{y}, \vec{v}) \mapsto \exp _{\infty \vec{y}}(\vec{v})$ uniformly in $(\vec{y}, \vec{v})$ as $n \rightarrow \infty$. In $B_{\delta^{\prime}}(\overrightarrow{0}) \times B_{\rho}(\overrightarrow{0})$, one has

$$
\left\|\exp _{n \vec{y}}(\vec{v})-\exp _{\infty \overrightarrow{0}}(\overrightarrow{0})\right\| \leq\left\|\exp _{n \vec{y}}(\vec{v})-\exp _{\infty \vec{y}}(\vec{v})\right\|+\left\|\exp _{\infty \vec{y}}(\vec{v})-\exp _{\infty \overrightarrow{0}}(\overrightarrow{0})\right\| .
$$

Moreover, fixing $\rho^{\prime}>0$, one can take $\epsilon>0$ such that $0<\rho^{\prime}-\epsilon<\rho^{\prime} / 2$, and find a pair $\rho, \delta^{\prime}>0$ such that for $(\vec{y}, \vec{v}) \in B_{\delta^{\prime}}(\overrightarrow{0}) \times B_{\rho}(\overrightarrow{0})$

$$
\left\|\exp _{\infty \overrightarrow{0}}(\overrightarrow{0})-\exp _{\infty \vec{y}}(\vec{v})\right\|<\rho^{\prime}-\epsilon,
$$

and a natural $N_{0}$ such that, on the same ball and for $n>N_{0}$,

$$
\left\|\exp _{n \vec{y}}(\vec{v})-\exp _{\infty \vec{y}}(\vec{v})\right\|<\rho^{\prime}-\epsilon .
$$


Therefore, in $B_{\delta^{\prime}}(\overrightarrow{0}) \times B_{\rho}(\overrightarrow{0})$, one has

$$
\left\|\exp _{n \vec{y}}(\vec{v})-\exp _{\infty \overrightarrow{0}}(\overrightarrow{0})\right\| \leq\left(\rho^{\prime}-\epsilon\right)+\left(\rho^{\prime}-\epsilon\right)<\rho^{\prime} .
$$

This is the thesis.

The items (c) and (d) are trivially proven by noticing that, as a consequence of the analogous property of the diffeomorphisms $F_{n}$ defined above, in the normal coordinates $\vec{y}$ and thus in any other coordinate system around $z \equiv \overrightarrow{0}$ which covers any set $N_{z i}$, the diffeomorphisms $\left(\vec{y}, \vec{y}^{\prime}\right) \mapsto \exp _{n \vec{y}}^{-1}\left(\overrightarrow{y^{\prime}}\right)$ converge to the function $\left.\left(\vec{y}, \vec{y}^{\prime}\right) \mapsto \exp _{\infty \vec{x}}^{-1}\left(\vec{y}^{\prime}\right)\right)$ uniformly with all the derivatives. Similarly, the geodesics $(\lambda, \vec{y}, \vec{v}) \mapsto \gamma_{n}(\lambda, \vec{y}, \vec{v})$ converge uniformly in all arguments jointly to the geodesic $(\lambda, \vec{y}, \vec{v}) \mapsto \gamma_{\infty}(\lambda, \vec{y}, \vec{v})$ with all the derivatives for $(\vec{y}, \vec{v}) \in B_{r}(\overrightarrow{0}) \times B_{r}(\overrightarrow{0})$. Employing our procedure to define the neighborhoods $\mathcal{N}_{z i}$ given above, it is possible to shrink them, maintaining all the relevant properties, in such a way that $\overline{\mathcal{N}}_{z i} \subset B_{r}(\overrightarrow{0}), i \in \mathbb{R}$. By consequence, as $n \rightarrow \infty$, the sequence of functions

$$
\left(\lambda, \vec{y}, \vec{y}^{\prime}\right) \mapsto \gamma_{n}\left(\lambda, y, y^{\prime}\right)=\vec{y}_{n}\left(\lambda, y, \exp _{n \vec{y}}^{-1}\left(\vec{y}^{\prime}\right)\right)
$$

defined on any set $[0,1] \times \overline{\mathcal{N}}_{z i} \times \overline{\mathcal{N}}_{z i}$, and the sequence of functions

$$
\sigma_{n}\left(y, y^{\prime}\right)=\mathbf{g}_{n}(\vec{y})\left(\exp _{n \vec{y}}^{-1}\left(\vec{y}^{\prime}\right), \exp _{n \vec{y}}^{-1}\left(\vec{y}^{\prime}\right)\right),
$$

defined on any set $\overline{\mathcal{N}}_{z i} \times \overline{\mathcal{N}}_{z i}$, converge uniformly in all the variables jointly, to the corresponding functions computed with respect to the metric $\mathbf{g}_{\infty}=\mathbf{g}$. Making a recurrent use of Lemma 2.2, this result can be proven also concerning the derivatives of any order in all variables and in any coordinate system.

Acknowledgement. I am particularly indebted to A. Cassa for his constant assistance in solving mathematical problems related to this work and for his numerous and always illuminating technical suggestions. It is a pleasure to thank I. G. Avramidi, E. Ballico, S. Delladio, F. Serra Cassano, A. Tognoli and R. M. Wald for helpful discussions and G. Esposito, B. S. Kay and D. Klemm for valuable suggestions. This work has been financially supported by a Postdoctoral Research Fellowship of the Department of Mathematics of the University of Trento.

\section{References}

[Av98] I. G. Avramidi, Covariant techniques for computation of the heat kernel, hep-th/9704166, Rev. Math. Phys. in press.

[BO86] M.R. Brown and A.C. Ottewill, Phys. Rev. D. 34, 1776 (1986).

[BEE96] J.K. Beem, P.E. Ehrlich, K.L. Easley, Global Lorentzian Geometry (Marcel Dekker, Inc., New York, 1996) 
[Br84] M.R. Brown, J. Math. Phys. 25, 136 (1984).

[BD82] N. D. Birrel and P. C. W. Davies, Quantum Fields in Curved Space (Cambridge University Press, Cambridge, 1982).

[Ca90] R. Camporesi, Phys. Rep. 196, 1 (1990).

[Ch84] I. Chavel, Eigenvalues in Riemannian Geometry (Academic Press, Inc., Orlando, USA, 1984).

[Da89] E. B. Davies, Heat Kernel and Spectral Theory (Cambridge University Press, Cambridge, 1989).

[dC92] M. P. do Carmo, Riemannian Geometry (Birkhäuser, Boston, 1992).

[E195] E. Elizalde, Ten Physical Applications of Spectral Zeta Functions (SpringerVerlag, Berlin, 1995).

[EORBZ94] E. Elizalde, S.D. Odintsov, A. Romeo, A.A. Bytsenko and S. Zerbini, Zeta Regularization Techniques with Applications (World Scientific, Singapore, 1994).

[EF97] R. Estrada and S.A. Fulling Distributional Asymptotic Expansions of Spectral Functions and the associated Green Kernels funct-an/9710003

[Fr75] F.G. Friedlander, The wave equation on a curved space-time (Cambridge University Press, Cambridge, 1975).

[Fu91] S.A. Fulling, Aspects of Quantum Field Theory in Curved Space-Time (Cambridge University Press, Cambridge, 1991).

[FR87] S.A. Fulling and S.N.M. Ruijsenaars, Phys. Rep. 152, 135 (1987).

[FSW78] S.A. Fulling, M. Sweeny, R. M. Wald, Commun. Math. Phys. 63, 257 (1978)

[Ga64] P. R. Garabedian, Partial Differential Equations (John Wiley and Sons, Inc., New York, 1964).

[Gi84] P.G. Gilkey, Invariance theory, the heat equation and the Atiyah-Singer index theorem Math. lecture series 11 (Publish or Perish Inc. Boston, Ma., 1984)

[KN63] S. Kobayashi and K. Nomizu, Foundations of Differential Geometry Vol.1 (Interscience Publishers, New York, 1963)

[Mo98a] V. Moretti, Commun. Math. Phys. 201, 327 (1999). 
[Mo98b] V. Moretti, One-loop stress-tensor renormalization in curved background: the relation between $\zeta$-function and point-splitting approaches, and an improved point-splitting procedure. UTM 540, gr-qc/9809006, J. Math. Phys. to appear

[Ru97] W. Rudin, Functional Analysis (TATA McGraw-Hill, New Delhi, (1997))

[RS80] M. Reed and B. Simon, Functional Analysis (Academic Press, London,1980).

[Sh87] M.A. Shubin, Pseudodifferential Operators and Spectral Theory (SpringerVerlag, Berlin, 1987)

[Ta96] M.E. Taylor Partial Differential Equations Vol II, (Springer, New York,(1996)).

[TO98] A. Tognoli, Approximation Theorems in real analytic and algebraic geometry in Lectures in Real Geometry, Ed. F. Broglia, Walter de Gruyter \& Co., Berlin-New York (1998).

[Wa78] R. M. Wald, Phys. Rev. D 17, 1477 (1978).

[Wa79] R. M. Wald, Commun. Math. Phys. 70, 226 (1979).

[Wa94] R.M. Wald, Quantum Field theory and Black Hole Thermodynamics in Curved Spacetime (The University of Chicago Press, Chicago, 1994). 